\title{
Membrane dynamics of -secretase provides a molecular basis for A binding and processing
}

\section{Somavarapu, Arun Kumar; Kepp, Kasper Planeta}

Published in:

ACS Chemical Neuroscience

Link to article, DOI:

10.1021/acschemneuro.7b00208

Publication date:

2017

Document Version

Peer reviewed version

Link back to DTU Orbit

Citation (APA):

Somavarapu, A. K., \& Kepp, K. P. (2017). Membrane dynamics of -secretase provides a molecular basis for A binding and processing. ACS Chemical Neuroscience, 8(11), 2424-2436.

https://doi.org/10.1021/acschemneuro.7b00208

\section{General rights}

Copyright and moral rights for the publications made accessible in the public portal are retained by the authors and/or other copyright owners and it is a condition of accessing publications that users recognise and abide by the legal requirements associated with these rights.

- Users may download and print one copy of any publication from the public portal for the purpose of private study or research.

- You may not further distribute the material or use it for any profit-making activity or commercial gain

- You may freely distribute the URL identifying the publication in the public portal

If you believe that this document breaches copyright please contact us providing details, and we will remove access to the work immediately and investigate your claim. 


\title{
Membrane dynamics of $\boldsymbol{\gamma}$-secretase provides a molecular basis for $\mathbf{A} \beta$ binding and processing
}

\author{
Arun Kumar Somavarapu and Kasper P. Kepp* \\ Technical University of Denmark, DTU Chemistry, DK-2800 Kongens Lyngby, Denmark. \\ * Corresponding Author E-mail: kpj@,kemi.dtu.dk Phone: +045 45252409
}

\begin{abstract}
$\gamma$-secretase produces $\beta$-amyloid (A $\beta$ ) within its presenilin (PS1) subunit, mutations in which cause Alzheimer's disease, and current therapies thus seek to modulate its activity. While the general structure is known from recent electron microscopy studies, direct loop- and membrane-interactions and explicit dynamics relevant to substrate processing remain unknown. We report a modeled structure utilizing the optimal multi-template information available, including loops and missing side chains, account of maturation cleavage, and explicit all-atom molecular dynamics in the membrane. We observe three distinct conformations of $\gamma$-secretase (open, semi-open, and closed) that remarkably differ by tilting of helix 2 and 3 of PS1, directly controlling active site availability. The large hydrophilic loop of PS1 where maturation occurs reveals a new helix segment that parallels the likely helix character of other substrates. The semi-open conformation consistently shows the best fit of $A \beta$ peptides, i.e. longer residence before release and by inference more trimming. In contrast, the closed, hydrophobic conformation is largely inactive and the open conformation is active but provides fewer optimal interactions and induces shorter residence time and by inference releases A $\beta$ peptides of longer lengths. Our simulations thus provide a molecular basis for substrate processing and changes in the $\mathrm{A} \beta_{42} / \mathrm{A} \beta_{40}$ ratio. Accordingly, selective binding to protect the semi-open "innocent" conformation provides a molecular recipe for effective $\gamma$-secretase modulators; we provide the full atomic structures for these states that may play a key role in developing selective $\gamma$-secretase modulators for treatment of Alzheimer's disease.
\end{abstract}

Keywords: $\gamma$-secretase; PSEN1; Alzheimer's Disease; conformation change; $\beta$-amyloid. 


\section{Introduction}

$\gamma$-secretase is a transmembrane protein complex comprised of four protein subunits: Nicastrin, presenilin 1 or 2 (PS1, PS2), anterior pharynx-defective 1 (APH1), and presenilin enhancer 2 (PEN2) ${ }^{1-}$

3. As an aspartyl protease it catalyzes intramembrane proteolysis of numerous type-1 membrane proteins, including the C-terminal C99 fragment ${ }^{4,5}$ of the amyloid precursor protein (APP), produced upon cleavage by BACE1 ( $\beta$-secretase), and the Notch receptor ${ }^{6}$. The first of these reactions involves sequential $\varepsilon$ - and $\gamma$-cleavages by PS, the catalytic subunit of $\gamma$-secretase, to produce the notorious $\beta$ amyloid $(A \beta)$ peptides of different lengths (37-49 residues) $)^{7,8}$. Among these, $A \beta_{40}$ and $A \beta_{42}$ are thought to play a key role in Alzheimer's disease $(A D)^{9}$, notably via their $A \beta_{42} / A \beta_{40}$ ratio ${ }^{10}$.

The mechanism whereby $\gamma$-secretase produces $A \beta$ peptides of variable length and toxicity is of considerable interest as a forefront of current drug development efforts ${ }^{6,11}$. Strict $\gamma$-secretase inhibitors such as semagacestat have shown adverse side effects in clinical trials ${ }^{12,13}$, partly due to lack of substrate specificity between APP and other substrates such as Notch ${ }^{6,14}$. Focus is moving towards modulators of $\mathrm{A} \beta$ production rather than strict inhibitors ${ }^{11,14,15}$.

Until recently, little was known of the detailed structure and dynamics except for the general transmembrane topology ${ }^{16,17}$, but many types of data together imply conformational modulation of distinct substrate orientations within the catalytic pocket ${ }^{18,19}$. A major step forward was the observations leading to the step-wise three-amino acid spaced trimming mechanism ${ }^{7,8,20,21}$ : The complex processes C99, after its guidance via nicastrin and PEN2 ${ }^{22}$, by two main pathways leading to $\mathrm{A} \beta_{49}$ or $A \beta_{48}$, followed by consecutive tri-amino acid spaced cleavage to produce shorter isoforms, dominated by $\mathrm{A} \beta_{40}$ or $\mathrm{A} \beta_{42}$ isoforms ${ }^{7}$. Another major step forward were the 3-dimensional structures using cryo-electron-microscopy at resolutions $3.4-4.4 \AA^{23-25}$. These structures revealed the intact topology of $\gamma$-secretase with 20 transmembrane (TM) helices $(1,9,7$, and 3 from nicastrin, presenilin 1 (PS1), APH-1, and PEN2 respectively). While nicastrin features a single TM helix and a large dynamic extracellular domain that probably helps to size-discriminate potential substrates ${ }^{26,27}$, APH-1 and PEN2 mostly traverse through the membrane to potentially modulate PS1 stability and endoproteolysis ${ }^{3}$. Notably, disorder in TM2 of PS1 was speculated to be mechanistically important ${ }^{24,28}$; the atomic motions producing this disorder are unknown.

Despite these efforts, multiple key features remain unknown: Most importantly, the large hydrophilic loop in PS1 (residues 273-374 from exon 8, 9 and 10) where autocatalytic cleavage occurs 
is missing in all reported structures. The structures indicate flexible regions of PS1, notably TM2, TM6, and the large hydrophilic loop. These particular dynamic parts may hold the secret to the peculiar substrate processing by $\gamma$-secretase ${ }^{29-31}$. Thus, the dynamics of $\gamma$-secretase with these parts included in a membrane is of major interest. Also, PS1 undergoes auto-endoproteolysis between TM6 and TM7, resulting in mature N-terminal (NTF) and C-terminal (CTF) fragments that are catalytically active, and with few exceptions this is required for function. Thus, modeling the mature, cleaved loop and its role in processing seems warranted ${ }^{32}$; previous simulations and experiments on PS1 suggests that this mature loop functions as a plug that, together with PEN2, controls cytosolic access to the active site $^{31,33}$, whereas nicastrin provides extracellular size control ${ }^{26}$.

We aimed to understand the full conformational dynamics of mature $\gamma$-secretase in the membrane with these features included. This required building a complete model using experimental constraints and templates for all subunits and additional multi-template modeling of missing residues and loops, the establishment of a realistic matured membrane-embedded system, and all-atom molecular dynamics (MD) in a physiologically relevant cellular environment (neutral $\mathrm{pH}$, physiological ion strength, all-atom protein and explicit solvent). The resulting model is in full accordance with known experimental structural data $(\sim 0.5-0.6 \AA$ RMSD) and provides the most complete $\gamma$-secretase structure so far described. We subjected this system to three 500 ns seeded MD simulations to explore the conformational ensemble of this system in atomic detail.

The simulations reveal three distinct conformational states of PS1 that explain the electron microscopy disorder in this subunit, while also providing insight into how the mature loop modulates these motions. Importantly, the three distinct conformations differ mainly in the C-terminal part of TM2 and N-terminal part of TM3 of PS1 very near the two catalytic aspartates. Recurrence of these states and the contacts preventing broader motions support the completeness and relevance of our identified motions, although the exact quantitative nature of these motions will depend on details of force fields and membrane model. Docking simulations for both $A \beta_{42}$ and $A \beta_{40}$ revealed that the semiopen conformation optimally fits the peptides, causes stronger binding and accordingly slower release and prolonged processing of peptides. We conclude that the semi-open conformation is the "innocent" and proficient active state of $\gamma$-secretase, whereas the closed and open conformations lead to inactivity or longer isoforms, respectively. Thus, our model provides a molecular basis for substrate processing by $\gamma$-secretase and indicates that the open conformation should be selectively targeted while the semiopen conformation should be preserved or enforced by structure-dynamics guided therapies. 


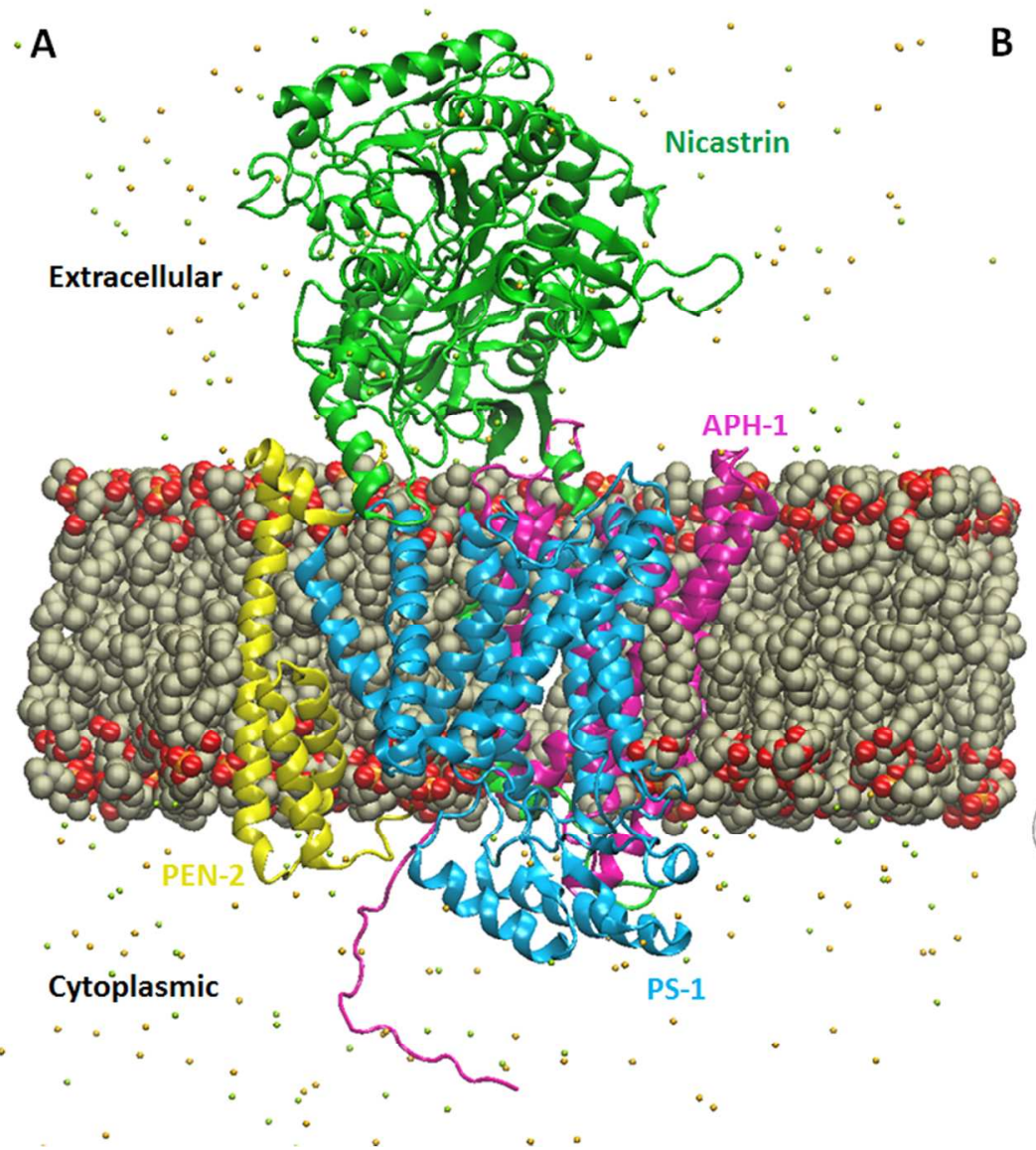

B

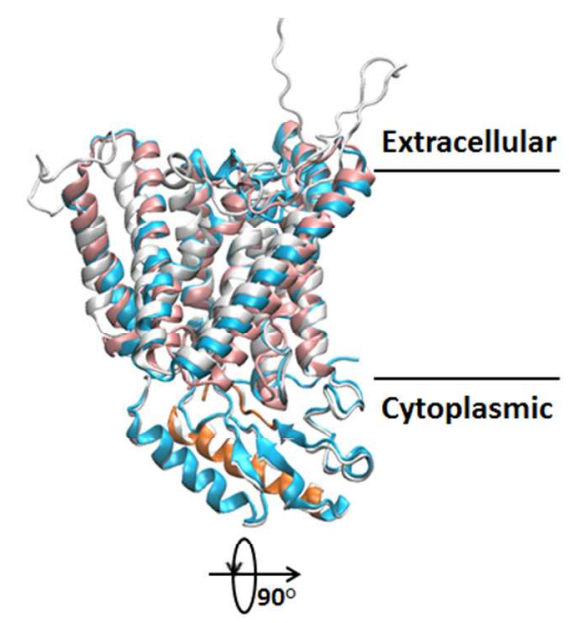

Figure 1. Structural model of membrane-bound $\gamma$-secretase. A) Simulated $\gamma$-secretase model with the four subunits Nicastrin (green), PS1 (cyan), APH-1 (magenta) and PEN-2 (yellow). Solvent and front row lipids were removed for better view. B) Two perpendicular views of multi-template PS1 (cyan) aligned with its templates 5FN3 (light pink) and our previously developed PS1 model ${ }^{31}$ (white) including both the exon 9 loop and hydrophilic loop 1.

\section{Results and Discussion}

\section{General dynamic structure of mature $\gamma$-secretase in a membrane}

As described above, despite major recent progress, the mechanistic understanding of $\gamma$-secretase seems to depend on the elusive, highly dynamic loop regions and disordered parts of the protein complex, including TM2 of PS1 very near the active site. Our multi-template "best of all worlds" model includes all complete transmembrane helices and the important loops from residues 71 to 467 for the first time, but also accounts for protonation states, realistic salt interactions, and the complex within the membrane. The model is shown in Figure 1A and 1B. The total RMSD measured between 5A63 and 
5FN3 experimental structures is $0.3 \AA$, and the total RMSD between our new $\gamma$-secretase model and $5 \mathrm{~A} 63$ or $5 \mathrm{FN} 3$ is 0.6 and $0.5 \AA$, i.e. within the thermal fluctuation range (RMSD plots in Figure S1 and S2, units of $\mathrm{nm}$ ) and thus in full agreement. The transmembrane topology is the most notable aspect of the general structure. The missing C-terminal residues added into Nicastrin (699-709) and APH-1 (245-265) were well converged during the MD simulations (Supporting Information, Figure S3) and attained stable conformations as seen from RMSD plots (Figure S2). However, the most important major movements and associated new insight relates to PS1 as discussed further below.

The three independent 500-ns ensembles provide a more complete estimate of the conformational variability in the general ensemble. The mature protein complex is a three-state system as seen from the principle component analysis, Figure S4. Though each simulation gave a distinct conformation from the last $200 \mathrm{~ns}$ (300-500 ns) used for clustering analysis, each also possesses other conformational states typical of the general ensemble that interconvert by crossing a relatively highenergy barrier. The RMSD plots (Figure S1) of the three trajectories cross at several time points, also indicating recurrence, which supports the qualitative completeness of the sampling and the importance of the identified helix motions.

Our model contains the large hydrophilic loop of PS1 (residues 273-374 from exons 8. 9, and 10) positioned towards the cytosol between TM6 and TM7. The important auto-endoproteolysis that produces the mature complex occurs within exon 9 (residues 290-319) ${ }^{34}$ and is included in our model by cleavage at residue 298 (Figure S5). Sequential auto-cleavage begins at residue 292/293, followed by cleavage at 295/296, and ending with $\gamma$-like cleavage at 298/299 similar to that seen for other substrates ${ }^{34-36}$. The details of this cleavage process is beyond the scope of this work as the in vivo size distribution and specific role of this trimming remains unclear, and we were mainly interested in enabling free loop motions of the mature state. Although additional trimming of the remaining residues is unlikely to affect the dynamics of the system, the different isoforms could modulate C99 cleavage by e.g. competitive binding, but this needs to be explored in future work.

Surprisingly, the large hydrophilic loop contains a loosely bound helix, a $\beta$-turn, and the remaining part as coil (the orange region in the bottom part of Figure 1B). These features are further enforced during simulation and are dynamically stable despite the sizable thermal fluctuations (Figure 2) that explain why these structures have been elusive. The helical segment is repeatedly obtained and is dynamically stable in two of the three simulations. Whereas this structure is unknown from any direct experimental information, our findings agree well with NMR data being consistent with both the 
presence of a $\beta$-turn and a loose $\alpha$-helix segment within this loop ${ }^{37}$. While we find substantial helix in the loop, the cleavage region 292-298 was not helical in our simulations. Thus this particular segment is disordered but may undergo helix transitions as it approaches the membrane-embedded active site. Following the dynamic "plug" movement of this loop ${ }^{31}$, auto-proteolysis is presumably achieved after docking of the larger helical loop segment including the cleavage site of immature PS1. This provides a notable parallel to $\mathrm{C} 99$, whose $\mathrm{A} \beta$ fragment adopts helical structures in micelles ${ }^{38-40}$ - this would imply a common structure-based mechanism for the trimming during maturation and C99 processing ${ }^{22,41}$.

PS1 cleaves APP-C99 and releases A $\beta$ peptides of varying length ranging from 37 to 49 residues $^{7,8}$. Current drug development efforts focus on this cleavage, although the binding modes and explicit molecular features that cause the differential cleavage are not known. The prevailing current hypothesis is that imprecise positioning of C99 leads to changes in the step-wise trimming of the peptide along two major paths leading to $A \beta_{40}$ and $A \beta_{42}$ respectively ${ }^{14,18,22}$.

It has been previously hypothesized that conformational flexibility within the catalytic subunit of PS1 affects cleavage ${ }^{24,29}$. Our previous PS1-alone simulations indicated that the membrane-packing of PS1 controls access to the active $\operatorname{site}^{31}$, consistent with more severe PSEN1 mutations in the membrane increasing the polarity of the protein and reducing its packing ${ }^{42}$. PCA on the last 200 ns of each trajectory produced the root-mean-square fluctuations (RMSF) for the first three eigenvectors (Figure 2). The most predominant motions reside in the large cytosolic hydrophilic loop region (273-374). Especially the residues 270-285, 317-328 and C-terminal of loop 360-373, where the structure is mostly coil, possess high RMSF values. These major motions resemble those seen for the isolated PS1 as described previously ${ }^{31}$; thus the major dynamics of free and complex-bound PS1 are qualitatively similar in this region. In addition, the loop (106-131) oriented towards the extracellular region and connecting TM1 and TM2 displays major motions, but the RMSF values are comparatively smaller than for free $\mathrm{PS}^{31}$, showing that the membrane-complex dampens these motions.

Interestingly, high fluctuations are also observed in residues 141-165, corresponding to the Cterminal end of TM2 (141-156), N-terminal end of TM3 (160-165) and a three-residue connecting loop. These residues lie directly opposite to the two catalytic aspartates D257 and D385 in TM6 and TM7, respectively, producing a cavity between them (Figure 3A). This cavity formed by TM2, TM3, TM5, TM6, and TM7 has been previously observed during sampling of PS1 conformational space through signal subtraction on cryo-EM image data and it was hypothesized to be the substrate binding region $^{24}$. 


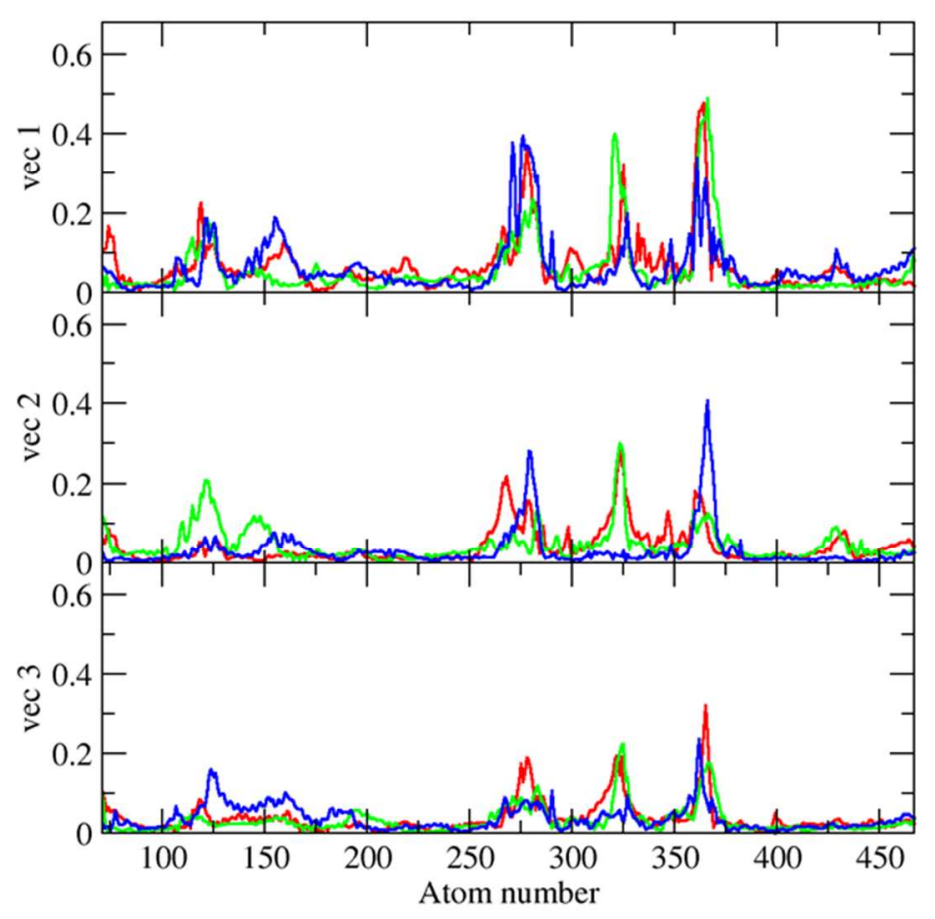

Figure 2. Dynamics of PS1 ensembles. Root-mean-square fluctuations of the first three Eigen vectors of PS1 within $\gamma$-secretase extracted from the full trajectory in the membrane (colors red, green and blue refer to three independent simulations). Major movements correspond to loop and TM2 and TM3 motions in PS1.

\section{Three distinct PS1 conformations of $\gamma$-secretase: Closed, semi-open, and open}

We examined the most representative structures obtained from clustering analysis of each $\gamma$-secretase simulation (Figure 3A) over the last 200 ns during which stable trajectories were observed (RMSD plots in Figure S1 and S2). Importantly, the three simulations visit three distinct conformation states, and these differences are almost exclusively occurring near the active site of PS1. State 1 represents a closed conformation of TM2 with most of TM2 (141-156) positioned close to D257 and D385. This "closed" state could plausibly exert steric control over any substrate binding, as explored further below. In state 2, TM2 obtains a distinctly more open conformation, whereas a small N-terminal part of TM3 (160-165) has moved towards the two catalytic aspartates; thus this conformation is referred to as "semi-open". The third state has both the C-terminal part of TM2 and the N-terminal part of TM3 in open conformations and is thus referred to as the (fully) "open" state. In Figure $\mathbf{3 A}$, the closed, semiopen and open states are shown in red, green and cyan color, respectively. The three conformational 
basins are evident from free energy analysis of the principle components (Figure S4), which makes mature (but not immature) PS1 a remarkably multi-state protein compared with most other globular proteins.

To quantify these conformational changes, we measured the average distance from the two catalytic aspartate residues (D257 and D385) to the C-terminal end of TM2 (141-156) (Figure 3B) and the N-terminal end of TM3 (160-165) (Figure 3C) during all three simulations. Initially, all three simulations started with an average distance of $18 \AA$ between the C-terminal end of TM2 and the catalytic aspartates, representing the "static" picture known from electron microscopy structures (in the cryo-electron microscopy structure 5FN3, this distance is $17.2 \AA$ ). However, during the last $200 \mathrm{~ns}$ when the distinct conformational states had manifested, the closed state displayed a distance of only $\sim 14 \AA$, whereas the other two states displayed distances of $\sim 19 \AA$. Interestingly, in the second simulation (in which the semi-open conformation was observed), we observed open-close-open transitions that represent breathing modes of the $\gamma$-secretase dynamics. The complex started as an open conformation and attained a closed state after approximately 10 ns. Gradually after 300 ns, it again reached an open conformation (Figure 3B). Likewise, the initial average distance between the Nterminal end of TM3 and the catalytic aspartates was $26 \AA$. While there is no significant difference in two of the simulations, the semi-open conformation has this distance contracted (21 $\AA$, Figure 3C).

As seen from the measured distances and from the general PS1 topology, TM3 is farther from the catalytic aspartates than TM2, and only six N-terminal residues showed substantial movements. Thus it seems implausible that TM3 alone can significantly affect substrate binding, but the interplay between its motion and the TM2 motion leads to a distinct semi-open state that not only reveals a general opening-closing process that is probably functionally important, but also directly affects substrate binding affinity, as investigated further below. The ensemble-averaged distances between the $\mathrm{C} \alpha$ atoms of the two catalytic aspartates are $10.7 \AA$ in the semi-open conformation, similar to the experimental $5 \mathrm{~A} 63$ structure $(10.8 \AA)^{25}$. The corresponding numbers for the open and closed states are 13.4 and 11.6 A (full ensemble fluctuations of this distance can be found in Figure S6). This distance is probably important for substrate cleavage precision and for the cleavage pathway initiation, and it is notable that the shortest distance coincides with a more compact, strong binding of peptides, as shown below. 


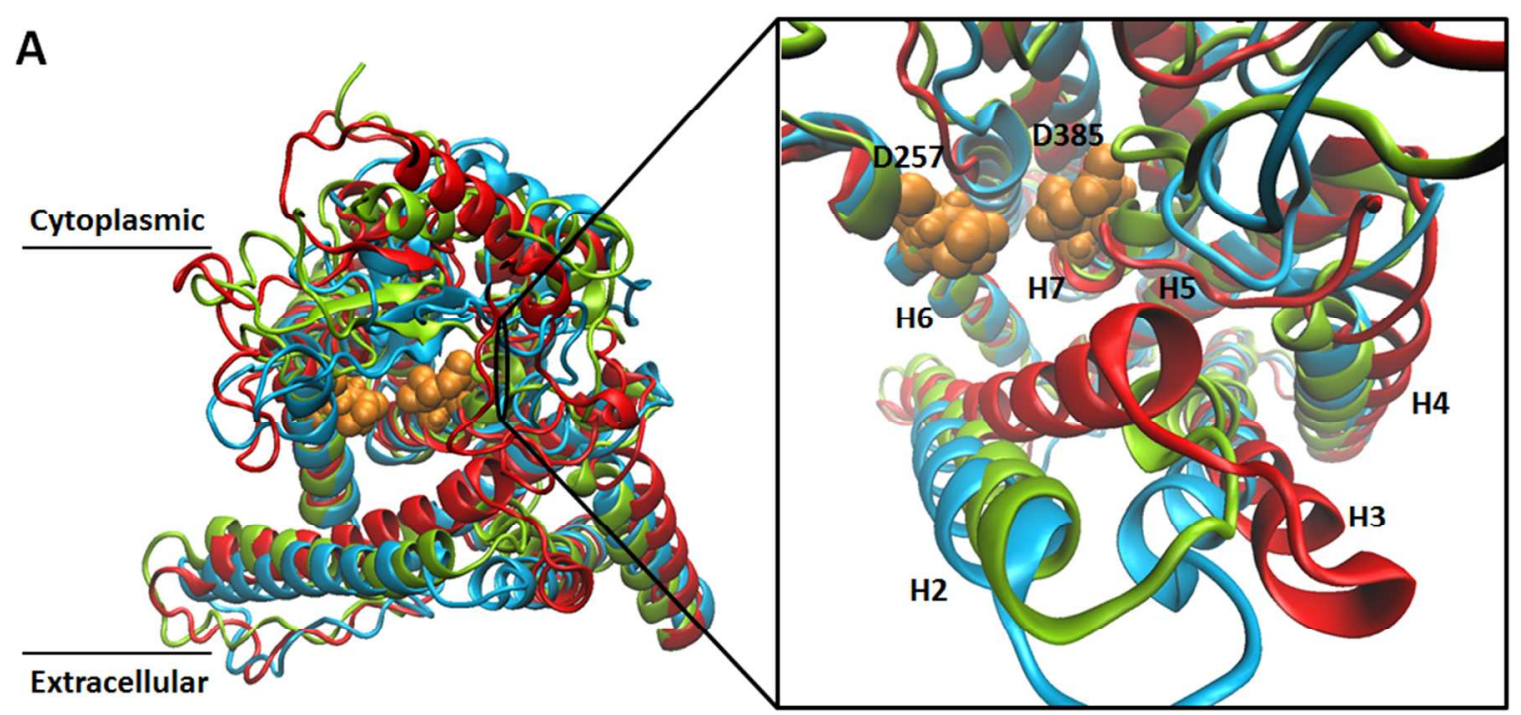

B

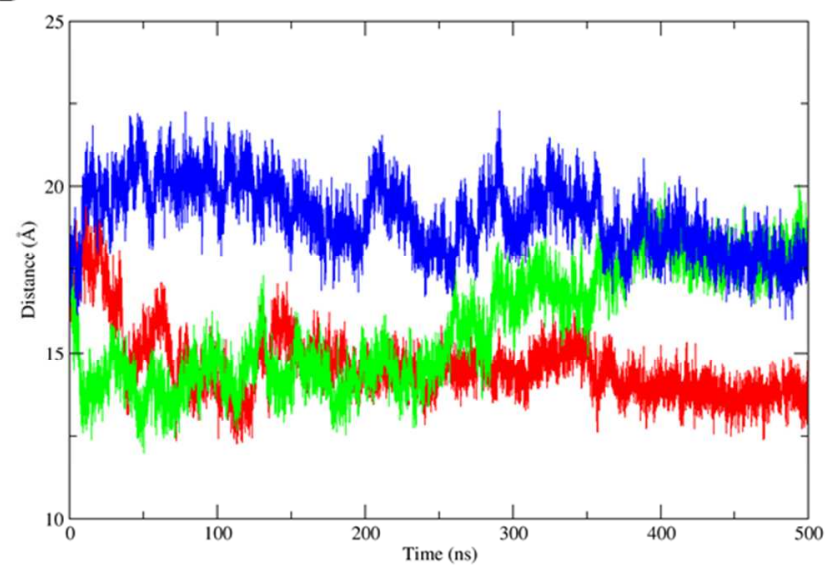

C

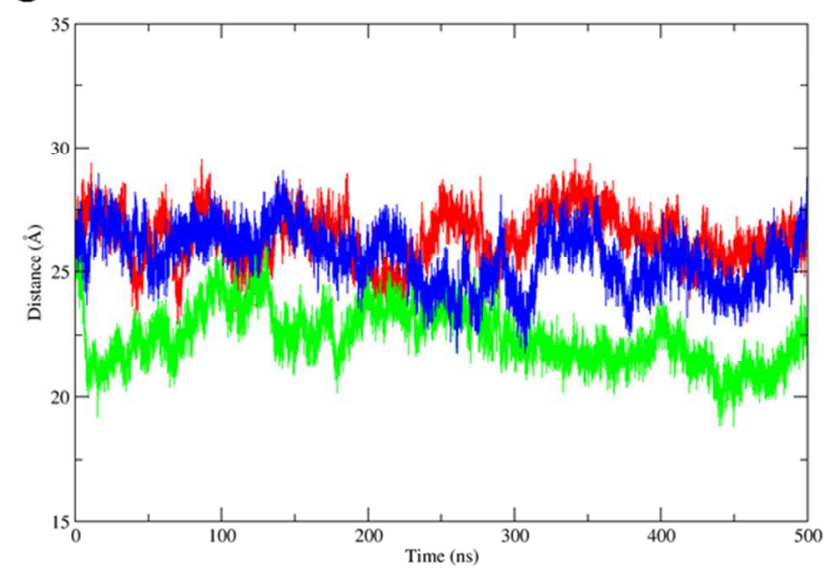

Figure 3. Conformational changes in PS1. A) Representative structures of PS1, extracted from three individual $\gamma$-secretase simulations superimposed, zoom-in image of active site shown for better view. The three PS1 conformations closed, semi-open and fully-open are shown in red, green and cyan respectively. B. Average distance calculated from catalytic aspartates to B) C-terminal end of TM2 (141-156) and C) N-terminal end of TM3 (160-165). The three time scale average distance plots in red, green and blue corresponds to closed, semi-open and fully open conformational states.

\section{Substrate binding to $\gamma$-secretase is controlled by conformation state}

As discussed above, the conformational dynamics affects the space within the substrate-binding region of $\gamma$-secretase. Once C99 is located within the protein complex, we hypothesized that the binding of different cleaved $A \beta$ peptide products would depend on the space and the interactions within the 
binding site. To understand this effect, we simulated the binding of both $A \beta_{42}$ and $A \beta_{40}$ to all three conformational states. Cleavage products of variable length differ in the C-terminal part that remains located within the substrate-binding site, whereas the similar N-terminal parts extend out to the extracellular side of the complex, and the two most prominent cleavage products were thus expected to probe differential binding well. To test sensitivity to protocol, we used both flexible (CABS-dock) and rigid (ZDOCK) docking programs. The CABS-dock program enables unbiased docking, which circumvents the problem of input bias, but requires smaller peptides for exhaustive searching, so we used $A \beta_{11-40}$ and $A \beta_{13-42}$ peptides for this method and compared it to the results obtained for the full peptides docked using ZDOCK.

Figure 4 shows the conformations produced by CABS-dock of the most representative pose of $A \beta_{11-40}$ (Figure 4A, $B$ and $\mathbf{C}$ ) and $A \beta_{13-42}$ (Figure 4D, $\mathbf{E}$ and $\mathbf{F}$ ) within the three representative structures of PS1. The structures with TM2 in a closed conformation (Figure 3A and 3B) are shown with docked peptides in Figure 4A and 4D; this conformation reduces the tendency of any substrate to bind near the active site, and thus we did not identify any conformation of $A \beta_{11-40}$ and $A \beta_{13-42}$ peptides near the catalytic aspartates. While the remaining two conformations display TM2 in an open conformation, the fully open conformation has the $\mathrm{C}$ - and N-terminal parts of TM2 and TM3 more open than the semi-open conformation. In the (fully) open state, excess space and lack of receptor contacts prevents binding of peptides near the catalytic aspartates (Figure 4C and 4F), implying that cleavage products of C99 are less strongly bound in this state. Interestingly, in the semi-open conformation the $\mathrm{C}$ - and N-terminal parts of TM2 and TM3 create optimal space for both A $\beta_{11-40}$ and $A \beta_{13-42}$ peptides to bind strongly near the catalytic aspartates (Figure 4B and 4E). Without prior knowledge of binding site, both $\mathrm{A} \beta$ peptides bind to the active site with the $\mathrm{C}$-terminal end towards the catalytic aspartates; this implies that we may have located the dominant peptide-bound conformation state of $\gamma$-secretase. The main receptor-peptide interactions resulting from binding to this active semiopen state are summarized in Table S1 (C-terminal part of $\left.\mathrm{A} \beta_{40}\right)$ and Table S2 (C-terminal part of $\left.\mathrm{A} \beta_{42}\right)$.

To test this further, we used another program, ZDOCK, which uses a rigid docking protocol to dock substrates, but enabling the use of full peptides. Figure 5 shows the top conformations of $\mathrm{A} \beta_{40}$ (1BA4) and $\mathrm{A} \beta_{42}$ (1IYT) NMR structures docked within the three top representative structures of PS1. Again, the closed TM2 conformation did not allow the A $\beta$ peptides to bind inside the substrate-binding region; instead they bound on the verge of TM2 towards the extracellular region (Figure 5A and 5D). 
This confirms the CABS results that we have identified the existence of a conformational state of $\gamma$ secretase that is inaccessible to the peptide C-terminals. ZDOCK confirms that the open and semi-open conformations enable docking of $A \beta$ peptides inside the substrate-binding region (Figure 5B, $\mathbf{C}, \mathbf{E}$ and F). We also took the advantage of an available NMR structure for $A \beta_{15-55}$ (PDB code: $2 \mathrm{LLM}$ ) and docked this structure into the three PS1 representative structures by using ZDOCK program. While the semi-open and open conformations of PS1 successfully accommodate $A \beta_{15-55}$ at the binding site, the closed conformation completely prevented the peptide from entering the active site (Figure S7).

Accordingly, we conclude that the semi-open state is the most active and produce shorter isoforms, the closed state will be inactive, and the open conformation will be less active and have a shorter residence time of substrates, implying earlier release of cleavage product, and, in an ensemble average, increased long-short ratios but lower total turnover. This particular phenotype of lower activity combined with longer isoform formation is commonly seen in genetic PS1 variants ${ }^{30,42-44}$.

Table 1. Top docking scores of $A \beta_{40}$ and $A \beta_{42}$ peptides docked into distinct protein conformations.

\begin{tabular}{lll}
\hline PS1 conformation & PDB code (length) & ZDOCK score \\
\hline closed & 1BA4 (1-40) & 1617 \\
closed & 1IYT (1-42) & 1830 \\
closed & 2LLM (15-55) & 2246 \\
semi-open & 1BA4 (1-40) & 1974 \\
semi-open & 1IYT (1-42) & 2072 \\
Semi-open & 2LLM (15-55) & 2570 \\
fully open & 1BA4 (1-40) & 1750 \\
fully open & 1IYT (1-42) & 1642 \\
fully open & 2LLM (15-55) & 2486 \\
\hline
\end{tabular}




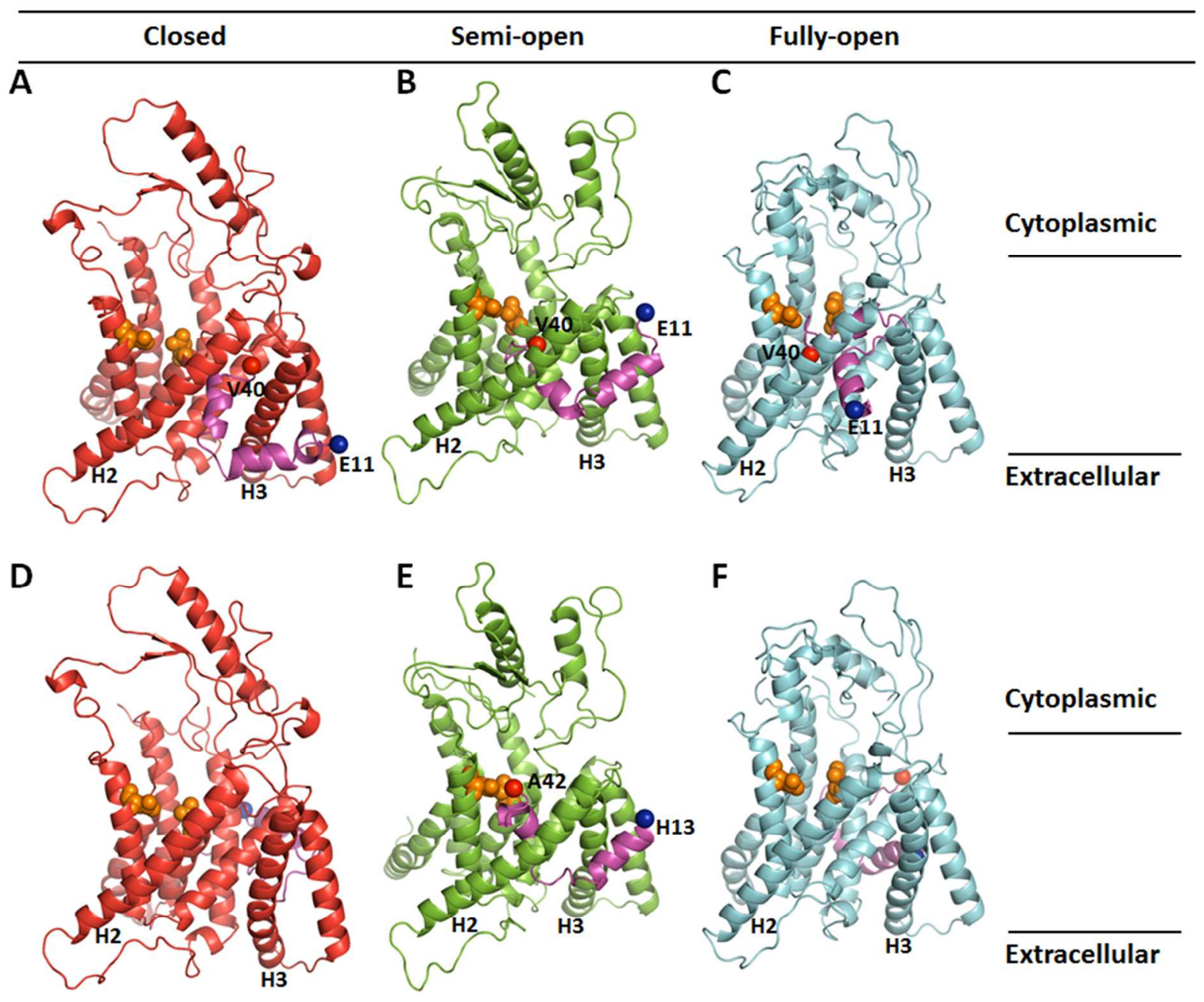

Figure 4. Molecular binding of A $\boldsymbol{\beta}$-type peptides to PS1. A-C) $A \beta_{11-40}$ and $\left.\mathbf{D}-\mathbf{F}\right) \mathrm{A} \beta_{13-42}$ peptides docked into the three representative structures of equilibrated PS1 within $\gamma$-secretase (red, green and cyan). $\mathrm{A} \beta$ peptides are colored in magenta with $\mathrm{N}$ - and $\mathrm{C}$-terminals shown as blue and red spheres. The two catalytic aspartates are shown as orange spheres.

The docking scores obtained from the ZDOCK program are collected in Table 1 and Table S3. The scores of the semi-open conformation are significantly higher (i.e. more favorable) than for the other two conformations, consistent with the finding from both complementary docking simulations that the semi-open conformation has optimal spatial constraints for receptor-peptide interactions. The results using both protocols thus support the same hypothesis that the semi-open conformation state provides the best fits of the $A \beta$ peptides. This state will, according to a simple model where binding affinity scales with substrate residence time, produce the longest residence time of C99 cleavage products and therefore, the most complete trimming by the two aspartates. 

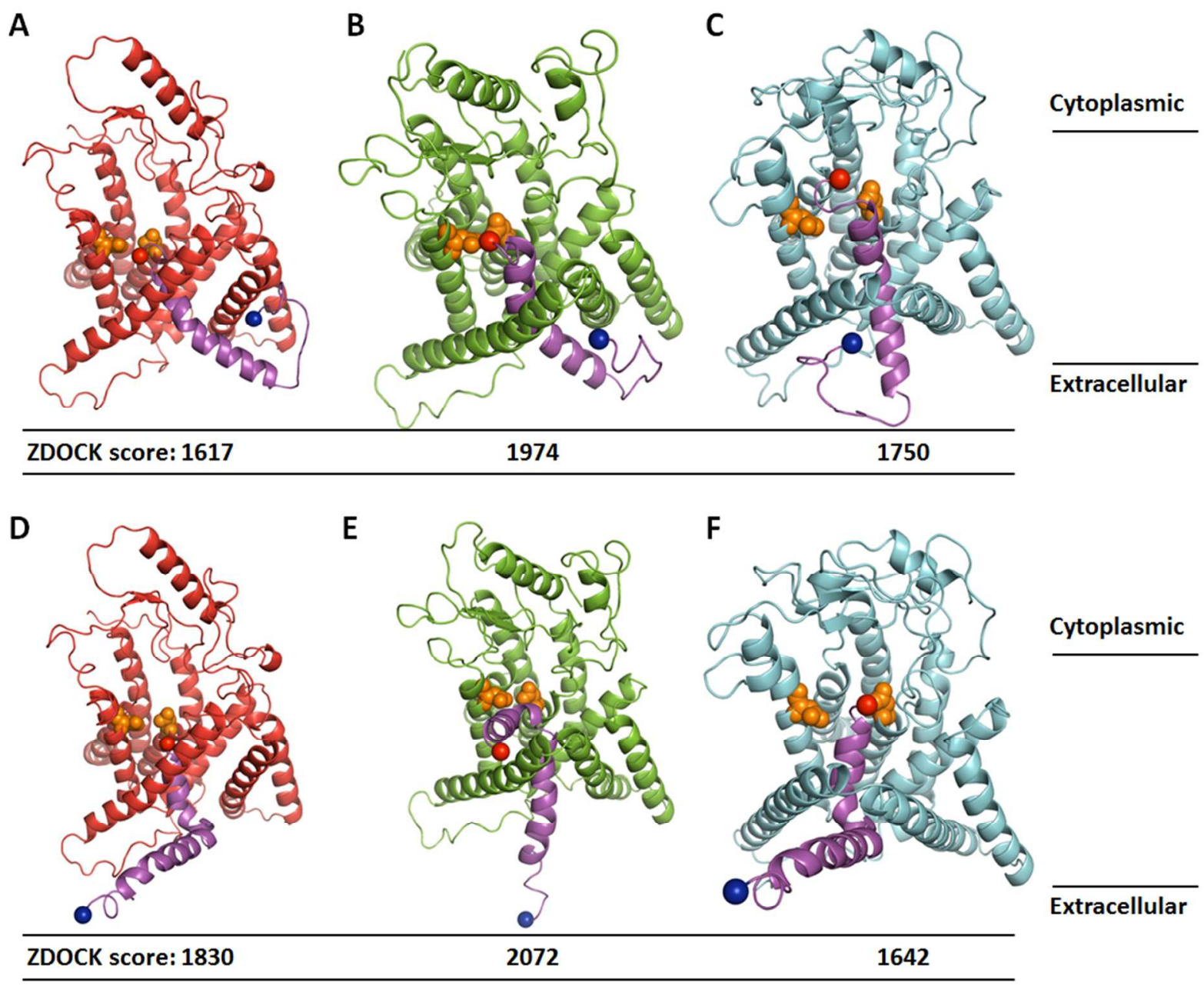

Figure 5. Binding poses of NMR structures of $\mathbf{A} \boldsymbol{\beta}$ to PS1. A-C) A $\beta_{40}$ (PDB ID: 1BA4) and D-F) $\mathrm{A} \beta_{42}$ (PDB ID: 1IYT) docked into the three representative structures of equilibrated PS1 within $\gamma$ secretase (red, green and cyan) using the ZDOCK program. A $\beta$ peptides are colored in magenta with $\mathrm{N}$ - and C-terminals shown as blue and red spheres. Catalytic aspartates are shown as orange spheres.

\section{Dynamics of substrate binding and processing}

As described above, we explored the complete conformational dynamics of mature $\gamma$-secretase, including trajectories of all atoms, side chains, loops and helices, except the 70 first loop residues of PS1, in the membrane at physiological ion strength and $\mathrm{pH}$ using three seeded 500 ns simulations. Interestingly, the ensemble structures from clustering analysis reveal three distinct conformations of PS1 differing in the C-terminal part of TM2 and the N-terminal part of TM3 near the catalytic 
aspartates. The open-close-open breathing dynamics of TM2 changes the space available in the active site (Figure 3A and Figure S8). While the C-terminal end of TM2 (141-156) and N-terminal end of TM3 (160-165) are directly connected by a short loop, movement of one region is hindered by the other and by nearby TM5, TM6 and TM7 helices. These motions are the major ones beyond loop motions, they recur repeatedly and interconvert, suggesting their primary relevance to the overall ensemble.

These conformational changes may have a direct effect on substrate processing. To support this hypothesis, two complementary types of docking simulations revealed that $\mathrm{A} \beta$ peptides bind substantially stronger in one conformation, the "semi-open" state (Figure 4B and 4E). Of the two conformations where A $\beta$ peptides could not fit the PS1 active site, one has TM2 in a closed conformation (Figure 4A and 4D) and the other has a too open conformation (Figure 4C and 4F).

The main explanations for altered $\gamma$-secretase processing relate to the impact of the active site topology, the orientation of the substrate within the cavity in several binding cavities, and the precision of cleavage ${ }^{18,19}$. Bolduc et al. ${ }^{18}$ found three binding pockets in PS1 that can accommodate three residues from substrates for successive tri-peptide cleavage; long-range motions specific to PS1 may explain why some mutations far from these pockets increase $A \beta_{42} / A \beta_{40}$ ratios ${ }^{31}$. From both studies, it emerges that tight substrate-enzyme interaction is needed for a precise cleavage. Since our identified semi-open conformation produces optimal spatial constraints for binding, we mapped the residues around our docked $A \beta_{15-55}$ peptide within $4 \AA$. Several aromatic residues (F, Y and $\left.\mathrm{W}\right)$ are arranged as a ladder, pointing their side chains towards the peptide. Y256 and F388 are close to the catalytic aspartates (Figure S9) and are likely constituents of the pocket observed by Bolduc et al. ${ }^{18}$

Although these features will affect the two general pathways leading to $A \beta_{40}$ and $A \beta_{42}$, our detailed all-atom dynamics and docking simulations point towards a previously overlooked mechanism that may critically determine trimming extent, namely residence time of the C99-derived peptide substrates during step-wise cleavage. This model is explained below:

Kinetic studies on enzymes and their transition states generally suggest that the active sites of enzymes are flexible and tend to close around substrates to maximize binding contacts ${ }^{45,46}$. Kinetic studies of wild type and FAD-related mutations of PS1 have revealed diverse effects on kinetic parameters $V_{\max }$ and $k_{\text {cat }}$. These experiments demonstrate that FAD mutants affect A $\beta$ cleavage in three different ways that include both loss of general catalytic activity and gain of toxic function ${ }^{47}$, most 
commonly in the form of increased $A \beta_{42} / A \beta_{40}$ ratios $^{42,48-50}$. Importantly, hypomorphic mutations with lower overall activities tend to increase the relative amount of longer isoforms ${ }^{42}$. In addition, intramembrane proteolysis by $\gamma$-secretase features $k_{\text {cat }}=0.0012 \mathrm{~s}^{-1}$, which is very low compared to soluble proteases $\left(>1 \mathrm{~s}^{-1}\right)^{51}$. A similarly low $k_{\text {cat }}$ of $0.0063 \pm 0.00021 \mathrm{~s}^{-1}$ was observed for rhomboid protease, another intramembrane protease involved in Parkinson's disease ${ }^{52}$. Accordingly, intramembrane proteolysis occurs on a time scale of minutes, and therefore a substrate must remain in the active site long enough to be cleaved ${ }^{41} \cdot \gamma$-secretase cleaves C99 at multiple sequential sites ${ }^{7,8}$, and thus stronger and longer substrate binding becomes a critical, overlooked feature of substrate trimming.

Our dynamic simulations and docking results show consistently that the conformations of the complex affect the substrate affinity differently: TM2 moves closer to the catalytic residues in the closed state to substantially reduce the substrate binding cavity formed by TM2, TM3, TM5, TM6 and TM7 (Figure 3A). As seen from the docking scores, this closed conformation cannot bind peptide products of the types docked, and the C99 peptide will be even less able to enter the substrate-binding region, causing reduced activity, i.e. the closed conformation represents a loss-of-function tendency of the ensemble (Figure 6A and 6D). The overall kinetic scheme representing these findings is shown in Figure S10.

In contrast, the other two states have TM2 in an open conformation. In the semi-open conformation, the N-terminal region of TM3 moved towards catalytic aspartates (D257 and D385) to provide optimal fits for continued processing of the substrates, and the affinity is consistently larger in this optimal-bind state (Table 1) and by inference, increases the rate of catalytic reaction (Figure 6B and 6E). This state thus represents the functional, optimal trimming state that we conclude should be preserved or enforced by molecular intervention strategies. In contrast, the (fully) open conformation has less optimal packing interactions and will thus keep peptides for shorter time and cause less trimming but also represents lower overall catalytic activity because of the generally reduced substrate affinity: This leads to premature dissociation of substrates which explains the generation of longer form of $\mathrm{A} \beta$ (Figure 6C and 6F). We thus identify this open conformation with the common "hypomorphic" PS1 mutant phenotype that increases long-short ratios. Out of two conformations where A $\beta$ peptides failed to dock in PS1 active site, one has TM2 in closed conformation (Figure 4A and 4D) and the other has it open (Figure 4C and 4F). 

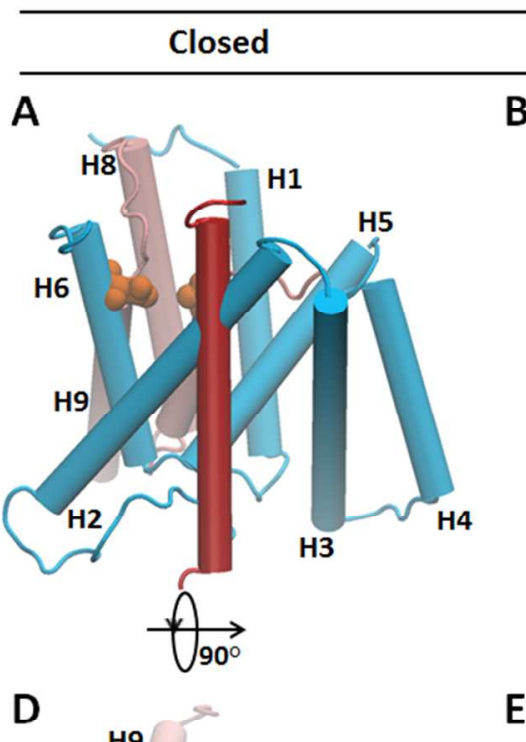

B

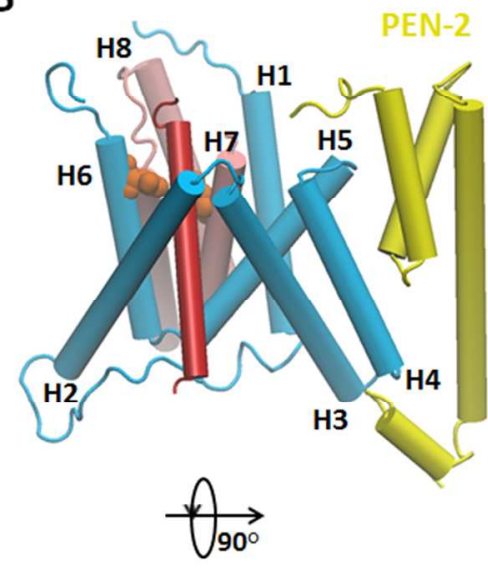

E
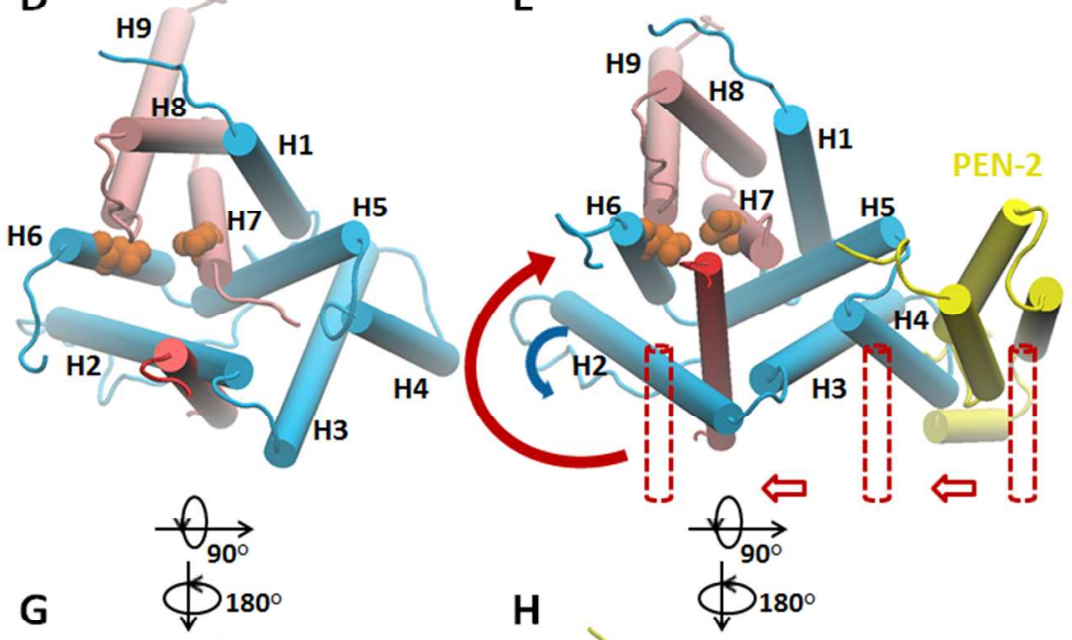

H
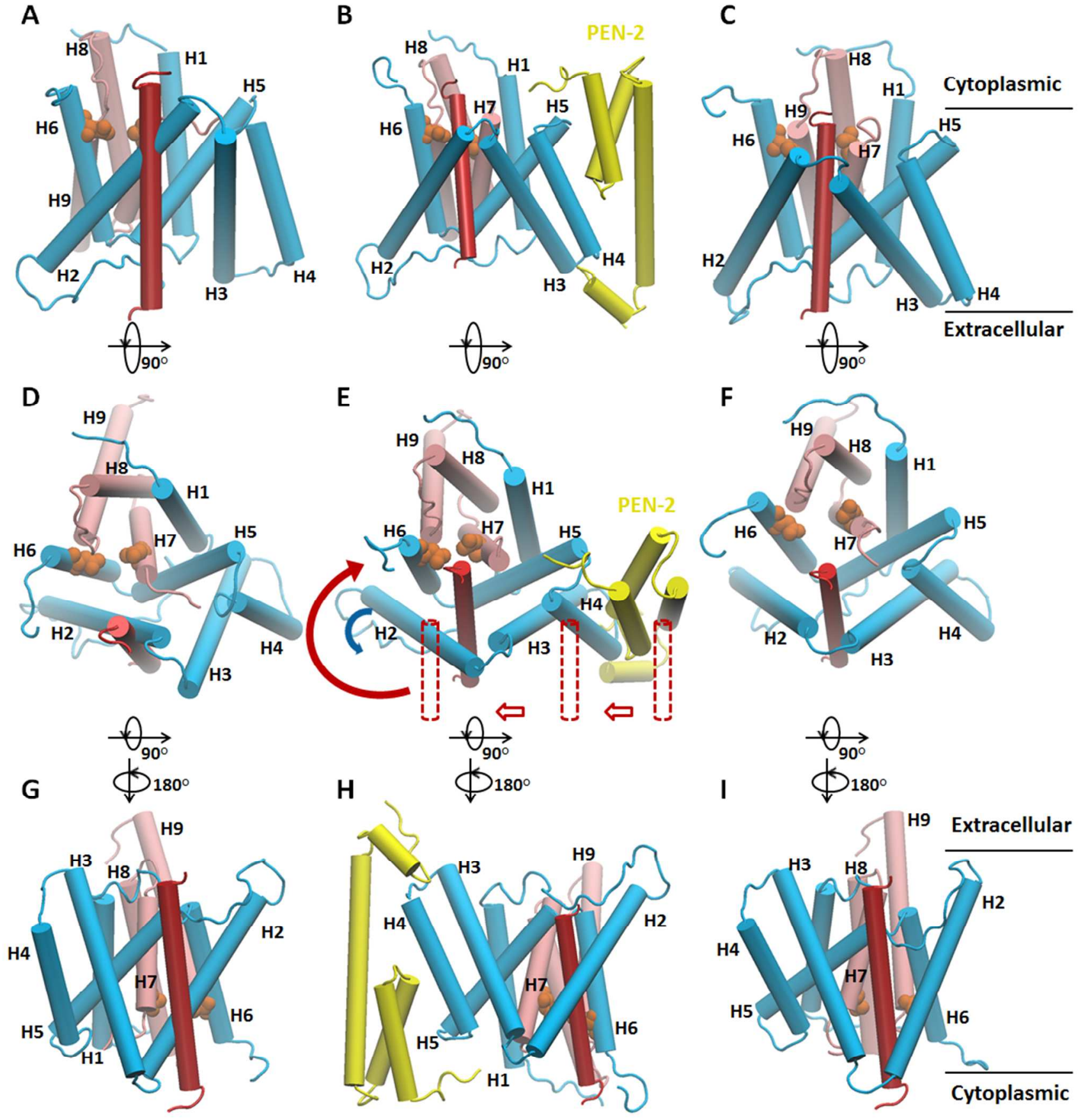

Figure 6. Model of C99 binding to conformations of PS1. Two perpendicular views of A, D) closed, B, E) semi-open and C, F) fully-open states of PS1 with $\mathrm{N}$ - and C-terminal fragments colored cyan and pink, respectively. The transmembrane region of C99 is shown as red cylinders, and the two catalytic aspartates as orange spheres. A, D) The closed conformation prevents substrate binding. B, E) The semi-open conformation maximizes substrate receptor contacts, residence time, and trimming. Stepwise translocation of C99 from PEN-2 ${ }^{22}$ implies a plausible C99 route into the semi-open 
conformation. The red arrow shows the entry route of C99 between TM2 and TM6 helices. The blue arrow depicts the outward movement of TM2. C, F) The open conformation displays excess space and insufficient substrate contacts, weaker binding, and earlier dissociation of presumably longer peptides (a phenotype commonly seen in FAD mutants). G-I) show the models in perspective with extracellular side upwards.

Although quantitative details will depend on the choice of force field, the sampling protocol, and the exact membrane system used, the observation of recurrence of the main states and the close contacts preventing broader motions of TM2 and TM3 support the completeness and relevance of these particular helix motions, and on this basis the proposed dynamic model of substrate processing seems qualitatively correct. It is notable that the "open" and "closed" conformations that we identify, which relate to TM2 and TM3 movement, active site space and substrate recruitment, are distinct from the previously proposed "open" and closed" states by Berezovska et al. ${ }^{53}$ which relate to the distance between N-terminal and C-terminal parts of the protein on the opposite side of the protein (Figure S11).

Recent photo-crosslinking studies by Fukumori et al. ${ }^{22}$ determined the N-terminal fragment of PS1 as the principal substrate-binding site. They also identified residues V44, L49, M51 and L52 from C99 as major $\gamma$-secretase interaction sites. In addition, they identified secondary substrate binding sites in PS1 NTF, Nicastrin and PEN-2 that aid substrate translocation towards the active site. Combining these findings of stepwise substrate recruitment with our dynamic structural model, Figure 6E shows the most plausible substrate recruitment route to reach the active site. During stepwise binding from PEN-2 to the active site, the loop regions between TM 2/3, 3/4 and 4/5 of PS1 NTF act as main barriers. A possible route where the connecting loop is absent is through TM2 and TM6. We showed in our previous PS1-alone study ${ }^{31}$ that TM2 moves outwards and thus potentially allows substrates to reach the active site through the TM2 and TM6 "doors". Interestingly, in GlpG, a Rhomboid-family intramembrane serine protease, TM5 bends outwards to provide access to substrates in a similar way ${ }^{54}$, and Chang et al. $^{55}$ showed reversible $\mathrm{pH}$-dependent open-close conformations in TM2 of transmembrane Bax inhibitor-1 protein, a calcium transporter with two aspartates located in a way similar to PS1, which has also been implied in calcium transport; we previously argued that calcium channel function and these motions seem coupled ${ }^{31}$. Also, the observed tendency that $\gamma$-secretase in thicker membranes produces less of the long $A \beta$ isoforms ${ }^{56}$ can be reconciled with our residence time 
model because thicker membranes favor the membrane-protein packing, tight C99 association and longer residence time, as described in our model.

Our model (Figure 6A-F) also provides a molecular structural basis for the processing phenotypes of PS1 mutations as discussed previously ${ }^{20}$. Different mutations are likely to affect one or more of these conformational states in specific ways, but the most common PS1 phenotype, which features loss of general function combined with increased long-short ratios, is characteristically obtained by favoring the open conformation of $\gamma$-secretase, whereas the semi-open "innocent" conformation is reduced in PS1 phenotypes. Our model is supported by the reduced dissociation rates of $A \beta_{42}$ from $\gamma$-secretase treated with $\gamma$-secretase modulators, and the increased dissociation rates of PS1 FAD mutants vs. wild type ${ }^{57}$, and agrees with chemoinformatics showing that severe PS1 mutations tend to reduce hydrophobicity more, and this correlates directly with higher $A \beta_{42} / A \beta_{40}$ $\operatorname{ratios}^{42}$, and explains why less proficient forms of the protein complex commonly produce relatively longer isoforms, because binding and effective trimming requires optimal hydrophobic and hydrophilic receptor-substrate contacts. Utilizing these conformations as targets for specific interventions can, we think, provide a molecular recipe of conformation-selective $\gamma$-secretase modulators as AD medicine ${ }^{41,58}$.

\section{Methods}

\section{Construction of atomic models}

Recently, multiple $\gamma$-secretase structures have been determined by cryo-electron microscopy (PDB codes: 4UIS, 5A63, 5FN2, 5FN3, 5FN4 and 5FN5) ${ }^{23-25}$. In order to utilize the maximum template coverage, we compared each $\gamma$-secretase subunit in all available experimental PDB structures. While a complete structure of PEN2 is available, C-terminal residue segments of length 11 and 21 were missing in Nicastrin and APH1, respectively (PDB codes: 5A63, 5FN2, 5FN3 and 5FN4). The catalytic subunit PS1 lacks several features: All available PS1 structures lack the large cytosolic hydrophilic loop region (residues 273-374 of exons 8, 9 and 10) where auto-proteolytic maturation occurs ${ }^{34}$. Also, TM2, which is situated close to the catalytic site and the extracellular hydrophilic loop 1 and has been implied as having a dynamic role in the protein ${ }^{25,31}$, is absent in the highest-resolution structure (5A63) at $3.4 \AA^{25}$. However these regions become well-ordered due to the binding of a kinked $\alpha$-helix from a co-purified protein in PDB structure 5FN3 at $4.1 \AA$ resolution ${ }^{24}$. 
To produce the most complete model possible, we used PS1 from PDBID 5FN3 ${ }^{24}$ and the PS1 model that we developed previously ${ }^{31}$, which is very similar in overall topology to the best-resolution structure of PS1 (5A63 at $3.4 \AA$ ) but importantly includes the large hydrophilic loop region and accounts for the high-resolution features of TM1-TM9. By using the two structures as templates, we generated a multi-template model using the MODELLER 9.14 software $^{59}$. Our new multi-template PS1 model uniquely covers all atoms from residues $71-467$, and misses only the $\mathrm{N}$-terminal 1-70 segment that is elusive in all experiments so far due to high disorder ${ }^{25}$.

After aligning the coordinates of our PS1 model onto the PS1 coordinates of 5FN3, we extracted the coordinates of all four subunits (including newly modeled PS1) into a single coordinate file; this produced a best-of-all-worlds model of the topology and helices while including our previously modeled and fully sampled loop regions that are experimentally undetermined. Structural constraints and bad contacts in the new integrated structure were reviewed and refined using the Protein Preparation Wizard tool of the Schrodinger suite ${ }^{60}$. Using the multi-chain model scripts available in MODELLER $9.14^{59}$, we added the missing C-terminal residues into Nicastrin (699-709) and APH1 (245-265) to generate a complete $\gamma$-secretase structural model, the most complete so far (Figure 1A). This model was then subject to MD simulations in an extensive membrane-solvent system to provide a realistic, physiologically relevant membrane environment for the protein, as described below.

We produced the mature model from the structurally optimized non-mature PS1 structure by separating the oxygen and nitrogen atoms of M298 and A299 at 6 A. Subsequently one oxygen atom and two hydrogen atoms were added to $\mathrm{CO}$ and $\mathrm{NH}$ groups of M298 and A299 respectively using Pymol to produce N-terminal (71-298) and C-terminal (299-467) fragments of mature PS1 (Figure 1B).

\section{Membrane simulation setup}

Although $\gamma$-secretase is a membrane protein complex, the available experimental structures do not feature the protein in the context of a membrane; the membrane is expected to affect substantially the protein dynamics as indicated by a previous membrane dynamics study of $\mathrm{PS}^{31}$. We therefore aimed to produce a realistic membrane model of the protein complex by embedding the new $\gamma$-secretase model into a membrane-solvent bilayer system (Figure 1A) using the membrane builder tool of the 
CHARMM-GUI web server ${ }^{61,62}$. We oriented the protein in membrane using the PPM (Position Protein in Membrane) server ${ }^{63}$. The output from the PPM server was used as input to the membrane builder tool. A homogeneous POPC lipid bilayer was generated around the protein by maintaining a water thickness of $12 \AA$ above and below the protein complex. Ion strength and salt impacts the conformations of proteins $^{64,65}$, and studying $\gamma$-secretase conformational changes with realistic salt conditions is thus necessary. To simulate a physiological ion concentration and to neutralize the -18 total charge of the complex, $0.15 \mathrm{M} \mathrm{NaCl}\left(165 \mathrm{Na}^{+}\right.$and $147 \mathrm{Cl}^{-}$ions $)$was placed using Monte-Carlo randomization.

MD simulations were performed using the GROMACS 5.0.4 package ${ }^{66,67}$ and the Charmm36 force field ${ }^{68}$, which is compatible with the membrane lipid parameters, and TIP3P ${ }^{69}$ as explicit water model. The Verlet ${ }^{70}$ cut-off scheme was used to treat non-bonded interactions with a cut-off for shortrange Coulomb and Lennard-Jones interactions of $12 \AA$. Long-range electrostatic interactions were calculated using the Particle Mesh Ewald ${ }^{71}$ algorithm. The Linear Constraint Solver (LINCS) algorithm $^{72}$ was used to constrain all covalent bond lengths. A steepest descent algorithm ${ }^{73}$ was applied to minimize the complete protein-lipid-solvent system, followed by six-step position-restrained equilibration for 375 ps to relax the atomic positions. During the first two steps, the system was equilibrated in a canonical (NVT) ensemble using Berendsen ${ }^{74}$ temperature coupling, and in the next four steps equilibration was achieved within the isothermal-isobaric (NPT) ensemble using the Berendsen $^{74}$ temperature and pressure couplings ${ }^{62}$. Finally, three independent 500-ns production simulations were carried out for the membrane-bound $\gamma$-secretase within an NPT ensemble using the Nose-Hoover thermostat ${ }^{75}$ and Parrinello-Rahman barostat $^{76}$ at $303.15 \mathrm{~K}$ and 1 atm, respectively.

\section{Principal component analysis (PCA)}

We used PCA to systematically reduce the dimensionality of our simulated data and extract underlying essential dynamics ${ }^{77}$. The correlations between the motions of particles produce the structure of total fluctuations. In a system of $\mathrm{N}$ atoms, $3 \mathrm{~N}-6$ modes of internal fluctuations are possible. The covariance between each pair of atoms is described by a matrix:

$$
C_{i j}=\left\langle\left(x_{i}-\left\langle x_{i}\right\rangle\right)\left(x_{j}-\left\langle x_{j}\right\rangle\right)\right\rangle
$$


Equation 1 represents the covariance matrix in which $x_{\mathrm{i}}$ and $x_{\mathrm{j}}$ are Cartesian coordinates of particles $i$ and $j$, respectively, and \langle\rangle is the ensemble average of all structures across the equilibrated (last $200 \mathrm{~ns}$ ) simulation time scale. Diagonalization of the symmetric covariance matrix $\boldsymbol{C}$ solves the eigenvalue problem,

$$
A^{T} C A=\lambda
$$

where $A$ represents the eigenvector and $\lambda$ is the associated eigenvalue. To focus on the catalytically relevant part of the protein complex, $\mathrm{C} \alpha$ atoms of the PS1 subunit were used in the covariance analysis. We applied the g_covar tool of the GROMACS package ${ }^{66,67}$ to calculate and diagonalize the covariance matrix. The Eigenvectors define the direction of collective motion of the atoms and the eigenvalues define how much total variance or motility is associated with each eigenvector; typically $>90 \%$ of the variance is described by $<10$ eigenvectors or principal components ${ }^{78}$. We used the g_anaeig tool of the GROMACS package ${ }^{66,67}$ to extract the most dominant fluctuations for further analysis.

\section{Clustering analysis}

To analyze the most frequently visited conformations of $\gamma$-secretase we performed ensemble-average clustering analysis by using the g_cluster tool of the GROMACS package ${ }^{66,67}$. The clustering algorithm is described by Daura et al ${ }^{79}$; we used a backbone root-mean-square deviation threshold of $2 \AA$. The last 200 ns of each simulation, which represent stable conformational ensembles as seen from RMSD plots (Supporting Information, Figure S1), were used in the clustering analysis. Based on the RMSD cut-off the algorithm groups all the structures into representative clusters of the ensemble and defines the structure with highest number of neighbor conformations as representative structure in each cluster $^{79}$.

\section{Peptide docking simulations}

Models of relevant parts of $\mathrm{C} 99$ and $\mathrm{A} \beta$ peptides were docked into the representative cluster structures of all three seeded simulations using the molecular docking simulation programs CABS-dock ${ }^{80,81}$ and ZDOCK 3.0.2 $2^{82,83}$, which complement each other well: Without prior knowledge of the binding site, CABS-dock uses flexible docking of the peptides into the protein. The program performs a binding site 
search by allowing full flexibility of the peptide and small fluctuations of the protein backbone, while keeping the protein structure near to the equilibrated conformation by a set of distance restraints. The docking protocol has been tested on a benchmark set of 103 bound and 68 unbound protein receptors ${ }^{81}$. The program selects the realistic conformations (poses) based on the K-medoids clustering method ${ }^{81}$. Due to the limitation in allowed peptide length of CABS-dock, the relevant C-terminal parts corresponding to peptides $A \beta_{11-40}$ and $A \beta_{13-42}$ were docked; the C-terminal is residing within the binding pocket whereas the N-terminal parts are outside in solution, so this is not likely to affect the substrate binding dynamics substantially. Considering the limitation in docking programs and the lack of knowledge regarding the $A \beta$ binding mode, we excluded the clusters where $A \beta$ peptides docked with the hydrophilic N-terminal inside the hydrophobic active site due to the high energy of such poses. To validate this protocol, we also used another program, ZDOCK, which is a rigid docking program which searches all possible binding modes between receptor protein and peptide in the translational and rotational space, and which can handle the full $A \beta$ peptides. Finally each pose is evaluated by an energy scoring function ${ }^{84}$. We provided the two catalytic aspartates D257 and D385 from PS1 and V36-A42 from $A \beta_{40-42}$ as binding site residues. Residues G37-A42 belong to the sequential tripeptide proteolytic cleavage region G37-T48/L49 of longer $A \beta$ forms $\left(A \beta_{48}\right.$ and $A \beta_{49}$ derived from $\varepsilon$ cleavage), which serve as suitable peptide binding residues near the catalytic aspartates ${ }^{7,8}$.

Acknowledgements. The Danish Council for Independent Research $\mid$ Natural sciences (FNU) is gratefully acknowledged for supporting this work (grant ID: DFF - 1323-00110B). The authors acknowledge support from the Danish Center for Scientific Computing (grant ID: 2012-02-23).

Author contributions. KPK designed the work. AKS constructed the models and performed the simulations. KPK and AKS analyzed the models and results and wrote the paper.

Supplementary information. Supplementary information includes representative structures (as pdb files) of the equilibrated models of $\gamma$-secretase complexes in the three conformation states, as well as a file with supplementary figures and data. 


\section{References.}

(1) Kimberly, W. T., LaVoie, M. J., Ostaszewski, B. L., Ye, W., Wolfe, M. S., and Selkoe, D. J. (2003) Gamma-secretase is a membrane protein complex comprised of presenilin, nicastrin, Aph-1, and Pen-2. Proc. Natl. Acad. Sci. U. S. A. 100, 6382-6387.

(2) Edbauer, D., Winkler, E., Regula, J. T., Pesold, B., Steiner, H., and Haass, C. (2003) Reconstitution of $\gamma$-secretase activity. Nat. Cell Biol. 5, 486-488.

(3) Takasugi, N., Tomita, T., Hayashi, I., Tsuruoka, M., Niimura, M., Takahashi, Y., Thinakaran, G., and Iwatsubo, T. (2003) The role of presenilin cofactors in the $\gamma$-secretase complex. Nature 422, 438441.

(4) Estus, S., Golde, T. E., Kunishita, T., Blades, D., Lowery, D., Eisen, M., Usiak, M., Qu, X. M., Tabira, T., and Greenberg, B. D. (1992) Potentially amyloidogenic, carboxyl-terminal derivatives of the amyloid protein precursor. Science 255, 726-8.

(5) Seubert, P., Vigo-Pelfrey, C., Esch, F., Lee, M., Dovey, H., Davis, D., Sinha, S., Schlossmacher, M., Whaley, J., and Swindlehurst, C. (1992) Isolation and quantification of soluble Alzheimer's betapeptide from biological fluids. Nature 359, 325-327.

(6) De Strooper, B., Iwatsubo, T., and Wolfe, M. S. (2012) Presenilins and $\gamma$-secretase: structure, function, and role in Alzheimer Disease. Cold Spring Harb. Perspect. Med. 2, a006304.

(7) Takami, M., Nagashima, Y., Sano, Y., Ishihara, S., Morishima-Kawashima, M., Funamoto, S., and Ihara, Y. (2009) $\gamma$-Secretase: successive tripeptide and tetrapeptide release from the transmembrane domain of $\beta$-carboxyl terminal fragment. J. Neurosci. 29, 13042-13052.

(8) Qi-Takahara, Y., Morishima-Kawashima, M., Tanimura, Y., Dolios, G., Hirotani, N., Horikoshi, Y., Kametani, F., Maeda, M., Saido, T. C., Wang, R., and Ihara, Y. (2005) Longer forms of amyloid beta protein: implications for the mechanism of intramembrane cleavage by gamma-secretase. J. Neurosci. $25,436-445$.

(9) Hardy, J., and Selkoe, D. J. (2002) The amyloid hypothesis of Alzheimer's disease: progress and problems on the road to therapeutics. Science 297, 353-356.

(10) Kuperstein, I., Broersen, K., Benilova, I., Rozenski, J., Jonckheere, W., Debulpaep, M., Vandersteen, A., Segers-Nolten, I., Van Der Werf, K., Subramaniam, V., Braeken, D., Callewaert, G., Bartic, C., D’Hooge, R., Martins, I. C., Rousseau, F., Schymkowitz, J., and De Strooper, B. (2010) Neurotoxicity of Alzheimer's disease A $\beta$ peptides is induced by small changes in the A $\beta 42$ to A $\beta 40$ ratio. EMBO J. 29, 3408-3420.

(11) Golde, T. E., Koo, E. H., Felsenstein, K. M., Osborne, B. A., and Miele, L. (2013) $\gamma$-Secretase 
inhibitors and modulators. Biochim. Biophys. Acta - Biomembr. 1828, 2898-2907.

(12) Doody, R. S., Raman, R., Farlow, M., Iwatsubo, T., Vellas, B., Joffe, S., Kieburtz, K., He, F., Sun, X., Thomas, R. G., Aisen, P. S., Siemers, E., Sethuraman, G., and Mohs, R. (2013) A phase 3 trial of semagacestat for treatment of Alzheimer's disease. N. Engl. J. Med. 369, 341-50.

(13) Karran, E., and Hardy, J. (2014) A critique of the drug discovery and phase 3 clinical programs targeting the amyloid hypothesis for Alzheimer disease. Ann. Neurol. 185-205.

(14) De Strooper, B., and Chávez Gutiérrez, L. (2015) Learning by Failing: Ideas and Concepts to Tackle $\gamma$-Secretases in Alzheimer's Disease and Beyond. Annu. Rev. Pharmacol. Toxicol. 55, 419-437. (15) De Strooper, B. (2014) Lessons from a Failed $\gamma$-Secretase Alzheimer Trial. Cell 159, 721-726. (16) Laudon, H., Hansson, E. M., Melén, K., Bergman, A., Farmery, M. R., Winblad, B., Lendahl, U., von Heijne, G., and Näslund, J. (2005) A nine-transmembrane domain topology for presenilin 1. J. Biol. Chem. 280, 35352-60.

(17) Wolfe, M. S. (2013) Toward the structure of presenilin/ $\gamma$-secretase and presenilin homologs. Biochim. Biophys. Acta - Biomembr. 1828, 2886-2897.

(18) Bolduc, D. M., Montagna, D. R., Seghers, M. C., Wolfe, M. S., and Selkoe, D. J. (2016) The amyloid-beta forming tripeptide cleavage mechanism of $\gamma$-secretase. Elife 5, e17578.

(19) Zoltowska, K. M., and Berezovska, O. (2017) Dynamic Nature of presenilin $1 / \gamma-S e c r e t a s e:$ Implication for Alzheimer's Disease Pathogenesis. Mol. Neurobiol. 1-10.

(20) Wolfe, M. S. (2012) Processive proteolysis by $\gamma$-secretase and the mechanism of Alzheimer's disease. Biol. Chem. 393, 899-905.

(21) Matsumura, N., Takami, M., Okochi, M., Wada-Kakuda, S., Fujiwara, H., Tagami, S., Funamoto, S., Ihara, Y., and Morishima-Kawashima, M. (2014) $\gamma$-Secretase Associated with Lipid Rafts: Multiple interactive pathways in the stepwise processing of $\beta$-carboxyl-terminal fragment. J. Biol. Chem. 289, 5109-5121.

(22) Fukumori, A., and Steiner, H. (2016) Substrate recruitment of $\gamma \square$ secretase and mechanism of clinical presenilin mutations revealed by photoaffinity mapping. EMBO J. e201694151.

(23) Sun, L., Zhao, L., Yang, G., Yan, C., Zhou, R., Zhou, X., Xie, T., Zhao, Y., Wu, S., Li, X., and Shi, Y. (2015) Structural basis of human $\gamma$-secretase assembly. Proc. Natl. Acad. Sci. U. S. A. 112, 6003-6008.

(24) Bai, X., Rajendra, E., Yang, G., Shi, Y., and Scheres, S. H. (2015) Sampling the conformational space of the catalytic subunit of human $\gamma$-secretase. Elife 4, 551-560.

(25) Bai, X., Yan, C., Yang, G., Lu, P., Sun, L., Zhou, R., Scheres, S. H. W., and Shi, Y. (2015) An 
atomic structure of human $\gamma$-secretase. Nature 525, 212-218.

(26) Bolduc, D. M., Montagna, D. R., Gu, Y., Selkoe, D. J., and Wolfe, M. S. (2016) Nicastrin functions to sterically hinder $\gamma$-secretase-substrate interactions driven by substrate transmembrane domain. Proc. Natl. Acad. Sci. 113, E509-E518.

(27) Shah, S., Lee, S.-F., Tabuchi, K., Hao, Y.-H., Yu, C., LaPlant, Q., Ball, H., Dann, C. E., Südhof, T., and Yu, G. (2005) Nicastrin Functions as a $\gamma$-Secretase-Substrate Receptor. Cell 122, 435-447. (28) Lu, P., Bai, X., Ma, D., Xie, T., Yan, C., Sun, L., Yang, G., Zhao, Y., Zhou, R., Scheres, S. H. W., and Shi, Y. (2014) Three-dimensional structure of human $\gamma$-secretase. Nature 512, 166-170. (29) Elad, N., De Strooper, B., Lismont, S., Hagen, W., Veugelen, S., Arimon, M., Horré, K., Berezovska, O., Sachse, C., and Chávez-Gutiérrez, L. (2015) The dynamic conformational landscape of $\gamma$-secretase. J Cell Sci 128, 589-598.

(30) Zoltowska, K. M., Maesako, M., and Berezovska, O. (2016) Interrelationship between Changes in the Amyloid $\beta$ 42/40 Ratio and Presenilin 1 Conformation. Mol. Med. 22, 329-337.

(31) Somavarapu, A. K., and Kepp, K. P. (2016) The dynamic mechanism of presenilin-1 function: Sensitive gate dynamics and loop unplugging control protein access. Neurobiol. Dis. 89, 147-156. (32) Thinakaran, G., Borchelt, D. R., Lee, M. K., Slunt, H. H., Spitzer, L., Kim, G., Ratovitsky, T., Davenport, F., Nordstedt, C., Seeger, M., Hardy, J., Levey, A. I., Gandy, S. E., Jenkins, N. A., Copeland, N. G., Price, D. L., and Sisodia, S. S. (1996) Endoproteolysis of presenilin 1 and accumulation of processed derivatives in vivo. Neuron 17, 181-90.

(33) Isoo, N., Sato, C., Miyashita, H., Shinohara, M., Takasugi, N., Morohashi, Y., Tsuji, S., Tomita, T., and Iwatsubo, T. (2007) A 442 Overproduction Associated with Structural Changes in the Catalytic Pore of $\gamma$-Secretase. J. Biol. Chem. 282, 12388-12396.

(34) Podlisny, M. B., Citron, M., Amarante, P., Sherrington, R., Xia, W., Zhang, J., Diehl, T., Levesque, G., Fraser, P., Haass, C., Koo, E. H., Seubert, P., St George-Hyslop, P., Teplow, D. B., and Selkoe, D. J. (1997) Presenilin proteins undergo heterogeneous endoproteolysis between Thr291 and Ala299 and occur as stable N- and C-terminal fragments in normal and Alzheimer brain tissue. Neurobiol. Dis. 3, 325-37. (35) Steiner, H., Romig, H., Grim, M. G., Philipp, U., Pesold, B., Citron, M., Baumeister, R., and Haass, C. (1999) The biological and pathological function of the presenilin-1 Deltaexon 9 mutation is independent of its defect to undergo proteolytic processing. J Biol Chem 274, 7615-7618. (36) Fukumori, A., Fluhrer, R., Steiner, H., and Haass, C. (2010) Three-amino acid spacing of presenilin endoproteolysis suggests a general stepwise cleavage of gamma-secretase-mediated 
intramembrane proteolysis. J. Neurosci. 30, 7853-7862.

(37) Knappenberger, K. S., Tian, G., Ye, X., Sobotka-Briner, C., Ghanekar, S. V., Greenberg, B. D., and Scott, C. W. (2004) Mechanism of ??-secretase cleavage activation: Is ??-secretase regulated through autoinhibition involving the presenilin-1 exon 9 loop? Biochemistry 43, 6208-6218.

(38) Coles, M., Bicknell, W., Watson, A. A., Fairlie, D. P., and Craik, D. J. (1998) Solution structure of amyloid beta-peptide(1-40) in a water-micelle environment. Biochemistry 37, 11064-77.

(39) Crescenzi, O., Tomaselli, S., Guerrini, R., Salvadori, S., D’Ursi, A. M., Temussi, P. A., and Picone, D. (2002) Solution structure of the Alzheimer amyloid $\beta$-peptide (1-42) in an apolar microenvironment: Similarity with a virus fusion domain. Eur. J. Biochem. 269, 5642-5648.

(40) Somavarapu, A. K., and Kepp, K. P. (2015) The Dependence of Amyloid-beta Dynamics on Protein Force Fields and Water Models. Chemphyschem 16, 3278-3289.

(41) Langosch, D., Scharnagl, C., Steiner, H., and Lemberg, M. K. (2015) Understanding intramembrane proteolysis: from protein dynamics to reaction kinetics. Trends Biochem. Sci. 40, 318327.

(42) Somavarapu, A. K., and Kepp, K. P. (2016) Loss of stability and hydrophobicity of presenilin 1 mutations causing Alzheimer's Disease. J. Neurochem. 137, 101-111.

(43) Kretner, B., Trambauer, J., Fukumori, A., Mielke, J., Kuhn, P., Kremmer, E., Giese, A., Lichtenthaler, S. F., Haass, C., Arzberger, T., and Steiner, H. (2016) Generation and deposition of A $\beta 43$ by the virtually inactive presenilin $\square$ 1 L435F mutant contradicts the presenilin loss $\square$ of $\square$ function hypothesis of Alzheimer's disease. EMBO Mol. Med. 8, 458-465.

(44) Quintero-Monzon, O., Martin, M. M., Fernandez, M. a., Cappello, C. a., Krzysiak, A. J., Osenkowski, P., and Wolfe, M. S. (2011) Dissociation between the processivity and total activity of $\gamma$ secretase: Implications for the mechanism of Alzheimer's disease-causing presenilin mutations.

Biochemistry 50, 9023-9035.

(45) Wolfenden, R. (1999) Conformational aspects of inhibitor design: enzyme-substrate interactions in the transition state. Bioorg. Med. Chem. 7, 647-652.

(46) Boehr, D. D., Nussinov, R., and Wright, P. E. (2009) The role of dynamic conformational ensembles in biomolecular recognition. Nat. Chem. Biol. 5, 789-796.

(47) Chávez-Gutiérrez, L., Bammens, L., Benilova, I., Vandersteen, A., Benurwar, M., Borgers, M., Lismont, S., Zhou, L., Van Cleynenbreugel, S., Esselmann, H., Wiltfang, J., Serneels, L., Karran, E., Gijsen, H., Schymkowitz, J., Rousseau, F., Broersen, K., and De Strooper, B. (2012) The mechanism of $\gamma$-Secretase dysfunction in familial Alzheimer disease. EMBO J. 31, 2261-2274. 
(48) Duff, K., Eckman, C., Zehr, C., Yu, X., Prada, C. M., Perez-tur, J., Hutton, M., Buee, L., Harigaya, Y., Yager, D., Morgan, D., Gordon, M. N., Holcomb, L., Refolo, L., Zenk, B., Hardy, J., and Younkin, S. (1996) Increased amyloid-beta42(43) in brains of mice expressing mutant presenilin 1. Nature 383, 710-713.

(49) Lemere, C. A., Lopera, F., Kosik, K. S., Lendon, C. L., Ossa, J., Saido, T. C., Yamaguchi, H., Ruiz, A., Martinez, A., Madrigal, L., Hincapie, L., Arango, J. C., Anthony, D. C., Koo, E. H., Goate, A. M., and Selkoe, D. J. (1996) The E280A presenilin 1 Alzheimer mutation produces increased A beta 42 deposition and severe cerebellar pathology. Nat Med 2, 1146-1150.

(50) Borchelt, D. R., Thinakaran, G., Eckman, C. B., Lee, M. K., Davenport, F., Ratovitsky, T., Prada, C.-M., Kim, G., Seekins, S., Yager, D., Slunt, H. H., Wang, R., Seeger, M., Levey, A. I., Gandy, S. E., Copeland, N. G., Jenkins, N. A., Price, D. L., Younkin, S. G., and Sisodia, S. S. (1996) Familial Alzheimer's Disease-Linked Presenilin 1 Variants Elevate A $\beta 1-42 / 1-40$ Ratio In Vitro and In Vivo. Neuron 17, 1005-1013.

(51) Kamp, F., Winkler, E., Trambauer, J., Ebke, A., Fluhrer, R., and Steiner, H. (2015) Intramembrane Proteolysis of $\beta$-Amyloid Precursor Protein by $\gamma$-Secretase Is an Unusually Slow Process. Biophys. J. $108,1229-1237$.

(52) Dickey, S. W., Baker, R. P., Cho, S., and Urban, S. (2013) Proteolysis inside the Membrane Is a Rate-Governed Reaction Not Driven by Substrate Affinity. Cell 155, 1270-1281. (53) Berezovska, O., Lleo, A., Herl, L. D., Frosch, M. P., Stern, E. a, Bacskai, B. J., and Hyman, B. T. (2005) Familial Alzheimer's disease presenilin 1 mutations cause alterations in the conformation of presenilin and interactions with amyloid precursor protein. J. Neurosci. 25, 3009-3017. (54) Wu, Z., Yan, N., Feng, L., Oberstein, A., Yan, H., Baker, R. P., Gu, L., Jeffrey, P. D., Urban, S., and Shi, Y. (2006) Structural analysis of a rhomboid family intramembrane protease reveals a gating mechanism for substrate entry. Nat. Struct. Mol. Biol. 13, 1084-91. (55) Chang, Y., Bruni, R., Kloss, B., Assur, Z., Kloppmann, E., Rost, B., Hendrickson, W. A., and Liu, Q. (2014) Structural basis for a pH-sensitive calcium leak across membranes. Science 344, 1131-1135. (56) Winkler, E., Kamp, F., Scheuring, J., Ebke, A., Fukumori, A., and Steiner, H. (2012) Generation of Alzheimer disease-associated amyloid $\beta 42 / 43$ peptide by $\gamma$-secretase can be inhibited directly by modulation of membrane thickness. J. Biol. Chem. 287, 21326-34.

(57) Okochi, M., Tagami, S., Yanagida, K., Takami, M., Kodama, T. S., Mori, K., Nakayama, T., Ihara, Y., and Takeda, M. (2013) $\gamma$-Secretase Modulators and Presenilin 1 Mutants Act Differently on Presenilin $/ \gamma$-Secretase Function to Cleave A $\beta 42$ and $\mathrm{A} \beta 43$. Cell Rep. 3, 42-51. 
(58) Fairlie, D. P., Tyndall, J. D., Reid, R. C., Wong, A. K., Abbenante, G., Scanlon, M. J., March, D. R., Bergman, D. A., Chai, C. L., and Burkett, B. A. (2000) Conformational selection of inhibitors and substrates by proteolytic enzymes: implications for drug design and polypeptide processing. J. Med. Chem. 43, 1271-81.

(59) Sali, A., and Blundell, T. L. (1993) Comparative protein modelling by satisfaction of spatial restraints. J. Mol. Biol. 234, 779-815.

(60) Madhavi Sastry, G., Adzhigirey, M., Day, T., Annabhimoju, R., and Sherman, W. (2013) Protein and ligand preparation: parameters, protocols, and influence on virtual screening enrichments. $J$. Comput. Aided. Mol. Des. 27, 221-234.

(61) Jo, S., Kim, T., Iyer, V. G., and Im, W. (2008) CHARMM-GUI: a web-based graphical user interface for CHARMM. J. Comput. Chem. 29, 1859-65.

(62) Jo, S., Kim, T., and Im, W. (2007) Automated builder and database of protein/membrane complexes for molecular dynamics simulations. PLoS One 2, e880.

(63) Lomize, M. A., Pogozheva, I. D., Joo, H., Mosberg, H. I., and Lomize, A. L. (2012) OPM database and PPM web server: resources for positioning of proteins in membranes. Nucleic Acids Res. 40, D370-D376.

(64) Zhang, Y., and Cremer, P. S. (2006) Interactions between macromolecules and ions: the Hofmeister series. Curr. Opin. Chem. Biol. 10, 658-663.

(65) Jensen, K. P. (2008) Improved interaction potentials for charged residues in proteins. J. Phys. Chem. B 112, 1820-7.

(66) Berendsen, H. J. C., van der Spoel, D., and van Drunen, R. (1995) GROMACS: A messagepassing parallel molecular dynamics implementation. Comput. Phys. Commun. 91, 43-56.

(67) Pronk, S., Páll, S., Schulz, R., Larsson, P., Bjelkmar, P., Apostolov, R., Shirts, M. R., Smith, J. C., Kasson, P. M., Van Der Spoel, D., Hess, B., and Lindahl, E. (2013) GROMACS 4.5: A highthroughput and highly parallel open source molecular simulation toolkit. Bioinformatics 29, 845-854. (68) Klauda, J. B., Venable, R. M., Freites, J. A., O’Connor, J. W., Tobias, D. J., Mondragon-Ramirez, C., Vorobyov, I., MacKerell, A. D., and Pastor, R. W. (2010) Update of the CHARMM All-Atom Additive Force Field for Lipids: Validation on Six Lipid Types. J. Phys. Chem. B 114, 7830-7843. (69) Jorgensen, W. L., Chandrasekhar, J., Madura, J. D., Impey, R. W., and Klein, M. L. (1983, July) Comparison of simple potential functions for simulating liquid water. J. Chem. Phys. AIP Publishing. (70) Verlet, L. (1967) Computer "experiments" on classical fluids. I. Thermodynamical properties of Lennard-Jones molecules. Phys. Rev. 159, 98-103. 
(71) Darden, T., York, D., and Pedersen, L. (1993) Particle mesh Ewald: An N $\log (\mathrm{N})$ method for Ewald sums in large systems. J. Chem. Phys. 98, 10089.

(72) Hess, B. (2008) P-LINCS: A parallel linear constraint solver for molecular simulation. J. Chem. Theory Comput. 4, 116-122.

(73) Fletcher, R., and Powell, M. J. D. (1963) A Rapidly Convergent Descent Method for Minimization. Comput. J. 6, 163-168.

(74) Berendsen, H. J. C., Postma, J. P. M., van Gunsteren, W. F., DiNola, A., and Haak, J. R. (1984) Molecular dynamics with coupling to an external bath. J. Chem. Phys. 81, 3684-3690.

(75) Nosé, S., and Klein, M. L. (1983) Constant pressure molecular dynamics for molecular systems. Mol. Phys. 50, 1055-1076.

(76) Parrinello, M. (1981) Polymorphic transitions in single crystals: A new molecular dynamics method. J. Appl. Phys. 52, 7182.

(77) Balsera, M. A., Wriggers, W., Oono, Y., and Schulten, K. (1996) Principal Component Analysis and Long Time Protein Dynamics. J. Phys. Chem. 100, 2567-2572.

(78) Ichiye, T., and Karplus, M. (1991) Collective motions in proteins: a covariance analysis of atomic fluctuations in molecular dynamics and normal mode simulations. Proteins 11, 205-17.

(79) Daura, X., Gademann, K., Jaun, B., Seebach, D., Van Gunsteren, W. F., and Mark, A. E. (1999) Peptide Folding: When Simulation Meets Experiment. Angew. Chemie Int. Ed. 38, 236-240.

(80) Kurcinski, M., Jamroz, M., Blaszczyk, M., Kolinski, A., and Kmiecik, S. (2015) CABS-dock web server for the flexible docking of peptides to proteins without prior knowledge of the binding site. Nucleic Acids Res. 43, W419-W424.

(81) Blaszczyk, M., Kurcinski, M., Kouza, M., Wieteska, L., Debinski, A., Kolinski, A., and Kmiecik, S. (2016) Modeling of protein-peptide interactions using the CABS-dock web server for binding site search and flexible docking. Methods 93, 72-83.

(82) Pierce, B. G., Wiehe, K., Hwang, H., Kim, B.-H., Vreven, T., and Weng, Z. (2014) ZDOCK server: interactive docking prediction of protein-protein complexes and symmetric multimers. Bioinformatics 30, 1771-1773.

(83) Pierce, B. G., Hourai, Y., and Weng, Z. (2011) Accelerating Protein Docking in ZDOCK Using an Advanced 3D Convolution Library. PLoS One (Keskin, O., Ed.) 6, e24657.

(84) Mintseris, J., Pierce, B., Wiehe, K., Anderson, R., Chen, R., and Weng, Z. (2007) Integrating statistical pair potentials into protein complex prediction. Proteins Struct. Funct. Bioinforma. 69, 511520. 
For Table of Contents Use Only

Membrane dynamics of $\boldsymbol{\gamma}$-secretase provides a molecular basis for A $\beta$ binding and processing Arun Kumar Somavarapu and Kasper P. Kepp*

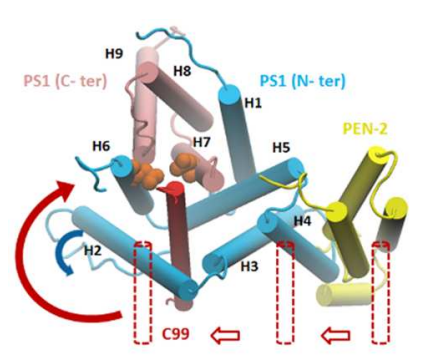

48

49

50

51

52

53

54

55

56

57

58

59

60 


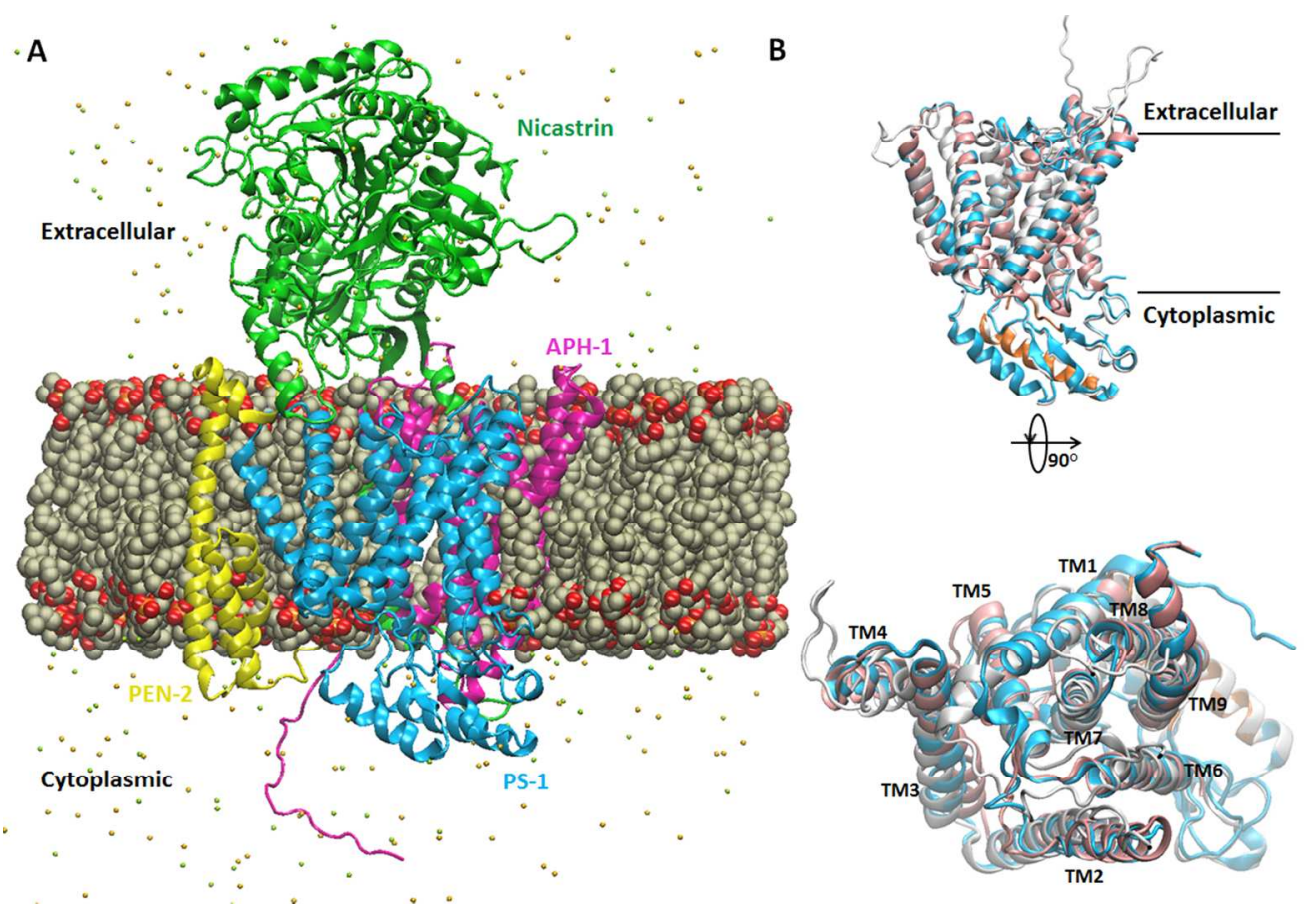

Figure 1

$101 \times 69 \mathrm{~mm}(300 \times 300$ DPI) 


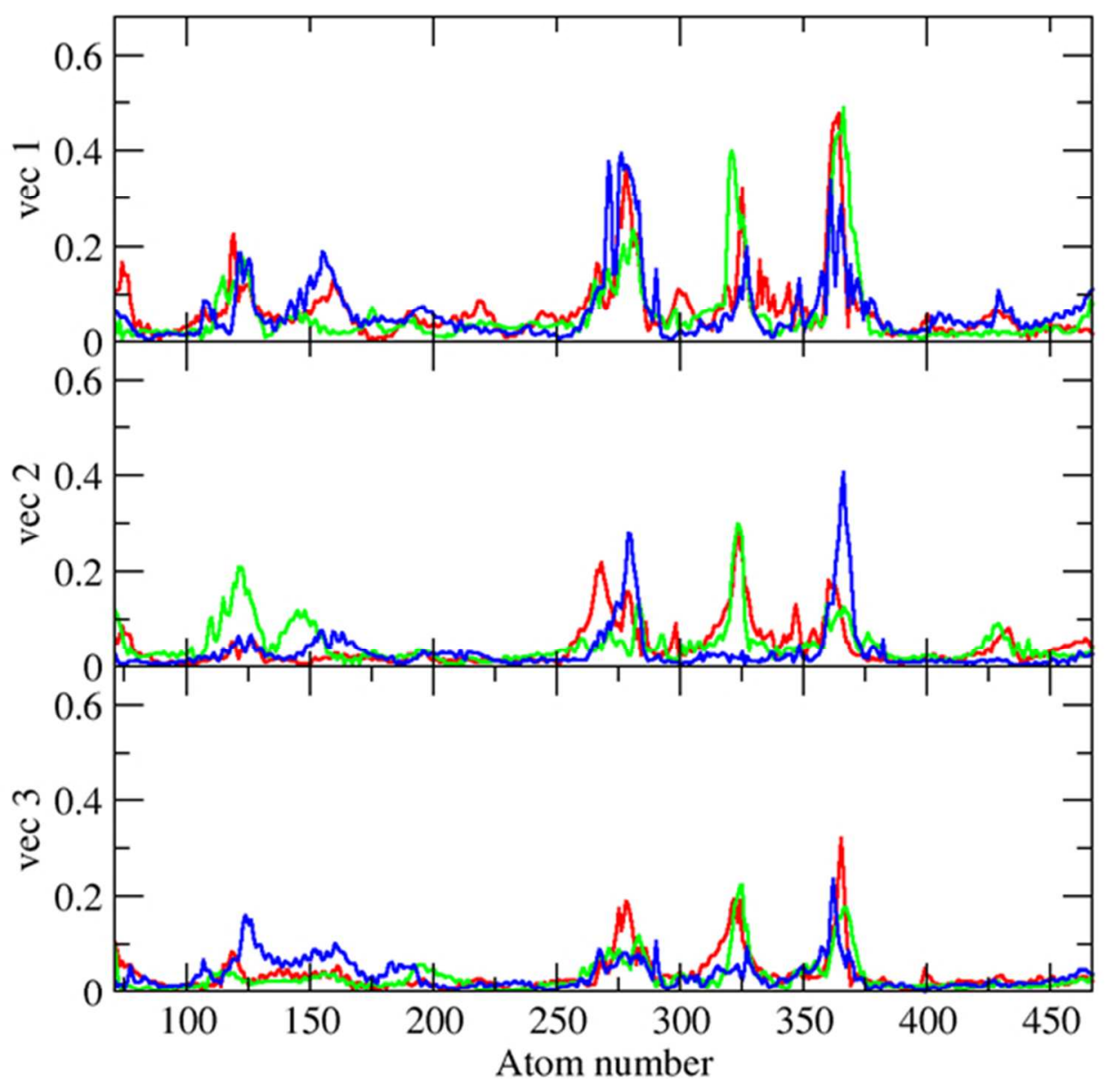

Figure 2

$55 \times 53 \mathrm{~mm}(300 \times 300$ DPI $)$ 


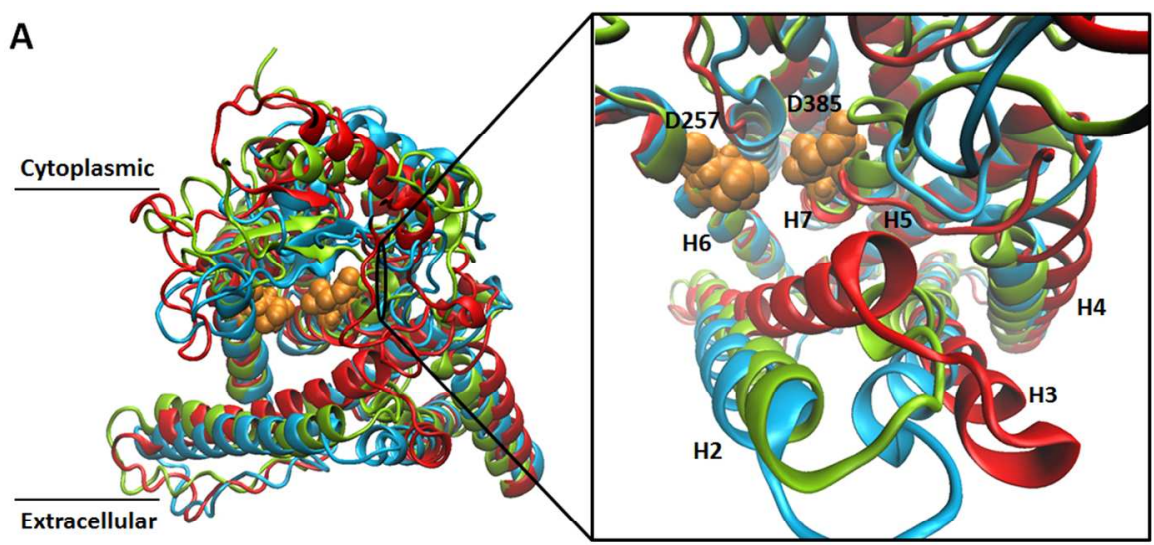

B

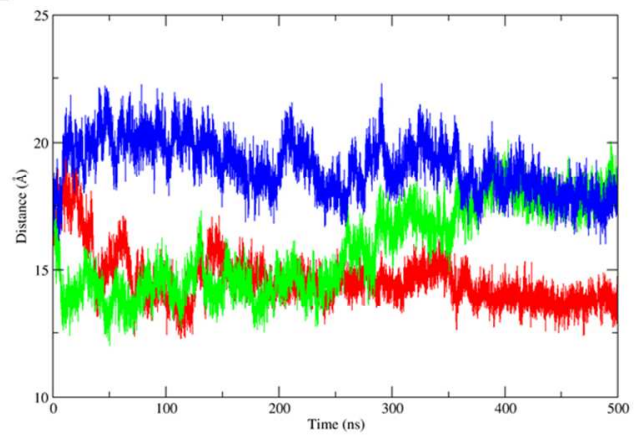

C

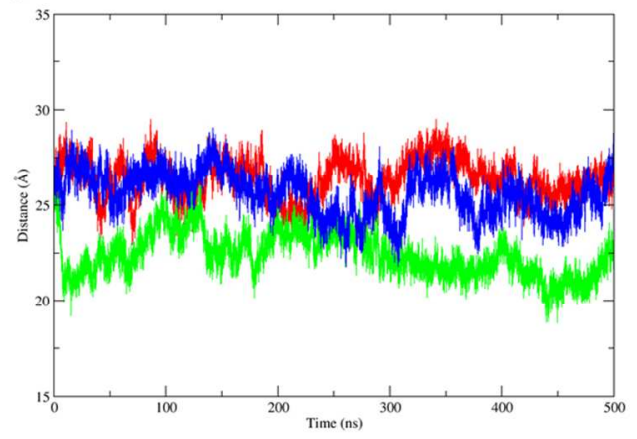

Figure 3

$100 \times 79 \mathrm{~mm}(300 \times 300 \mathrm{DPI})$ 


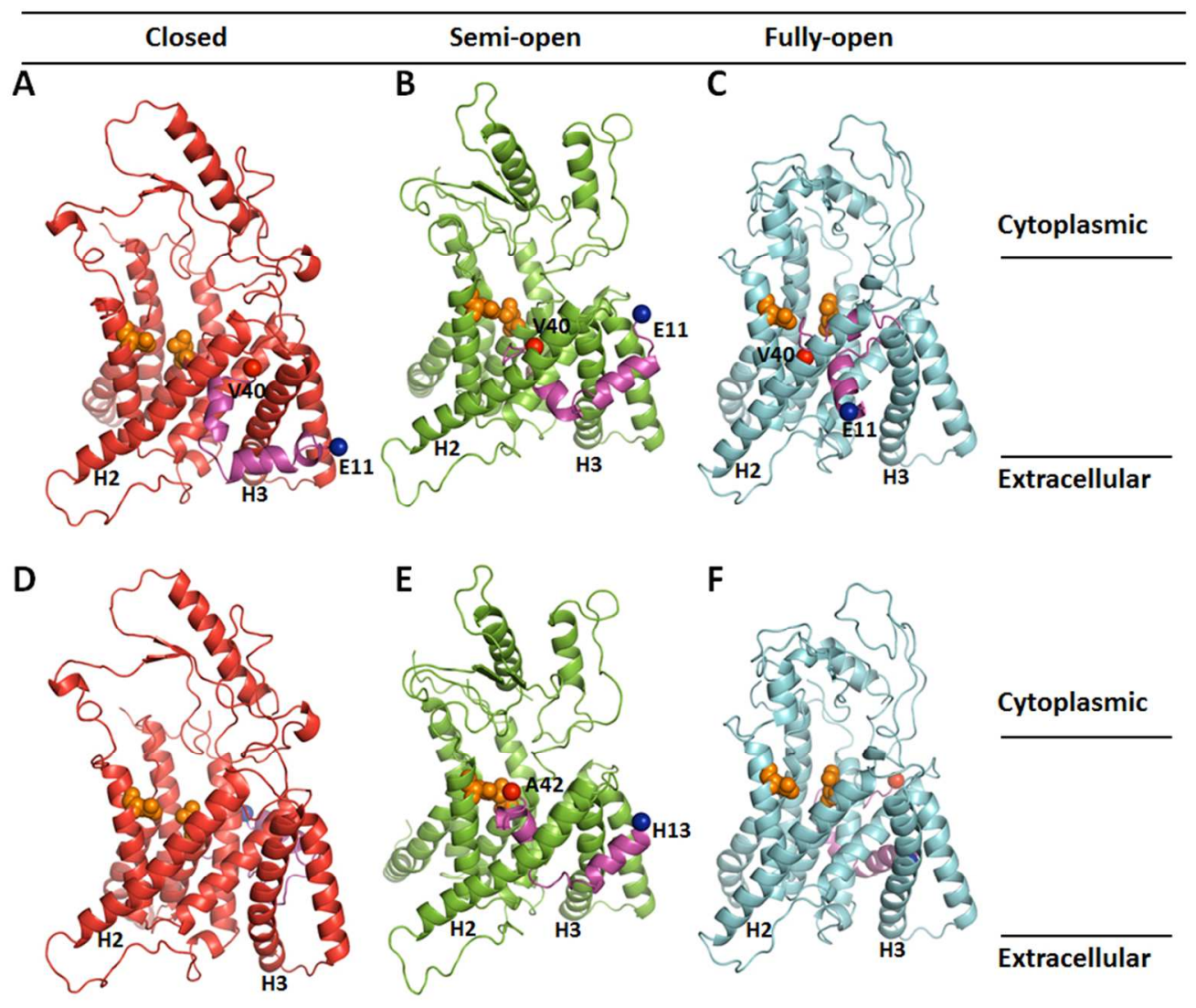

Figure 4

$81 \times 68 \mathrm{~mm}(300 \times 300$ DPI $)$ 


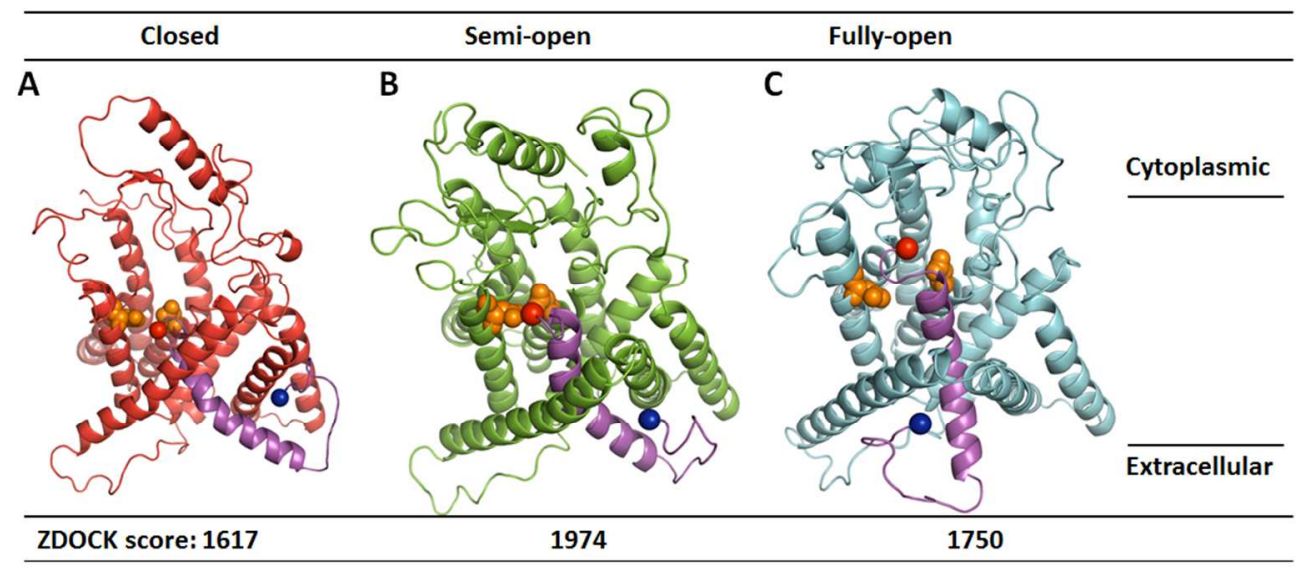

D

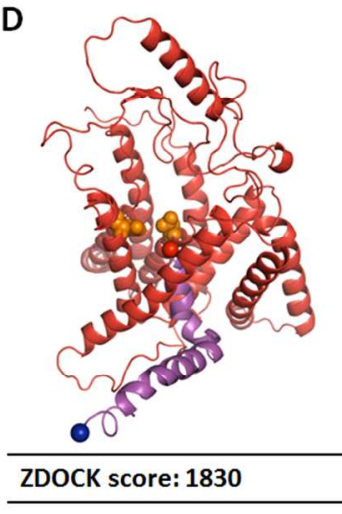

E

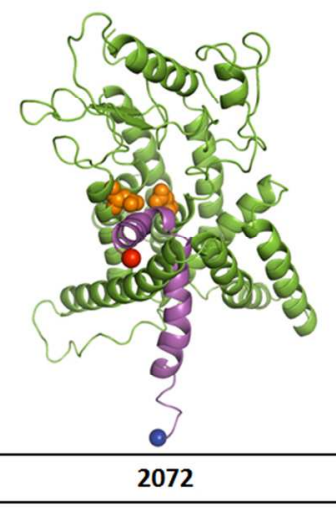

F

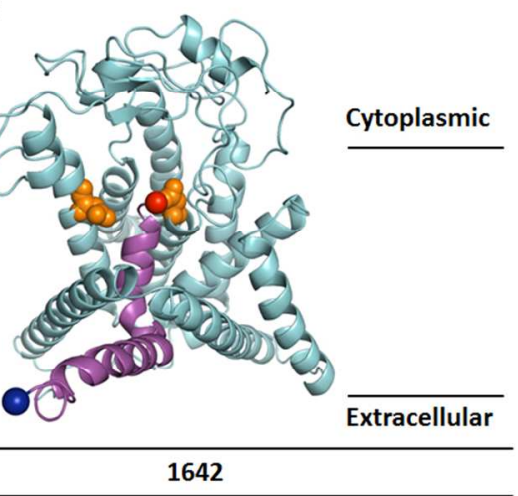

Figure 5

$93 \times 79 m m(300 \times 300$ DPI $)$ 


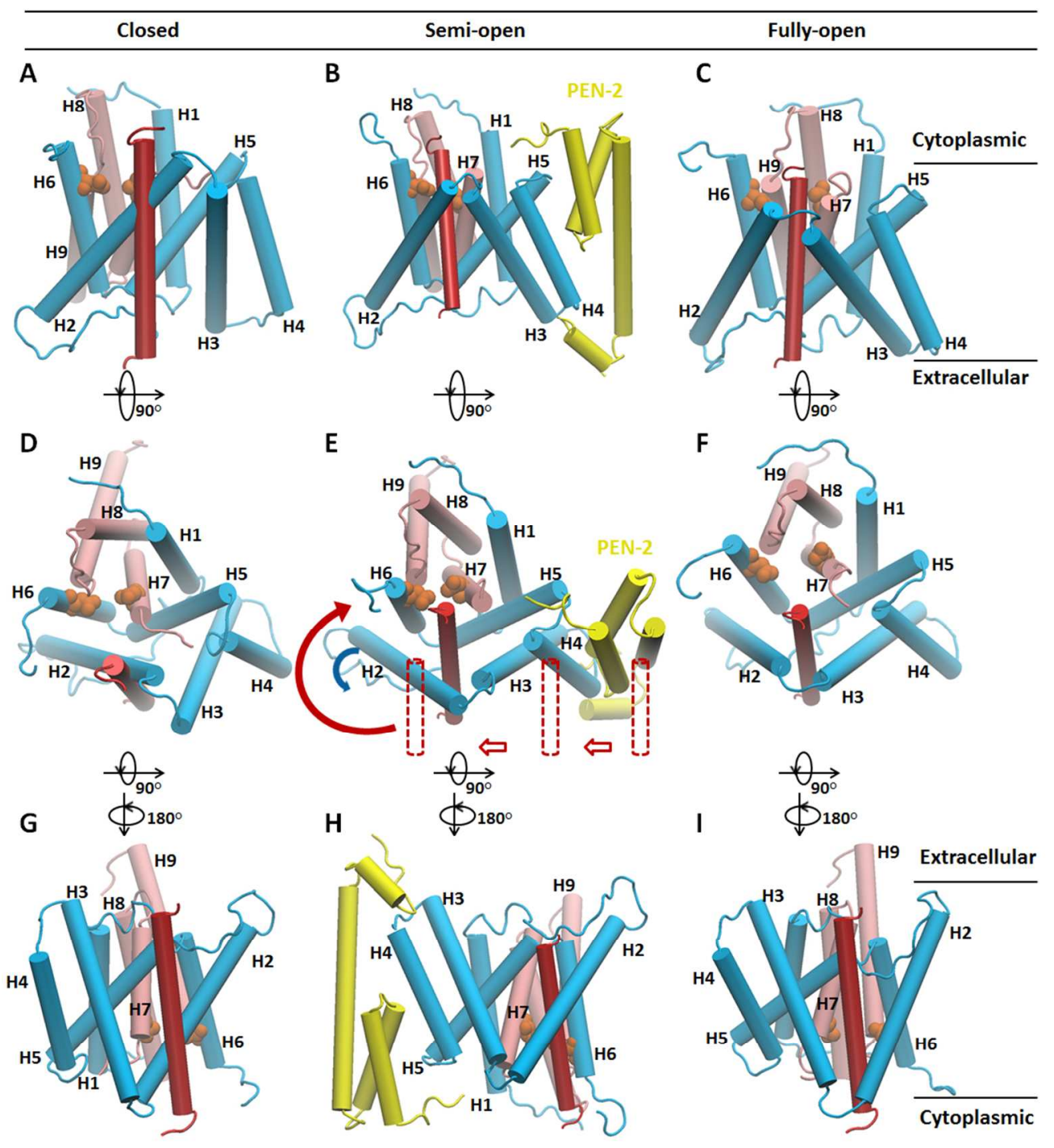

Figure 6

$94 \times 102 \mathrm{~mm}(300 \times 300 \mathrm{DPI})$ 


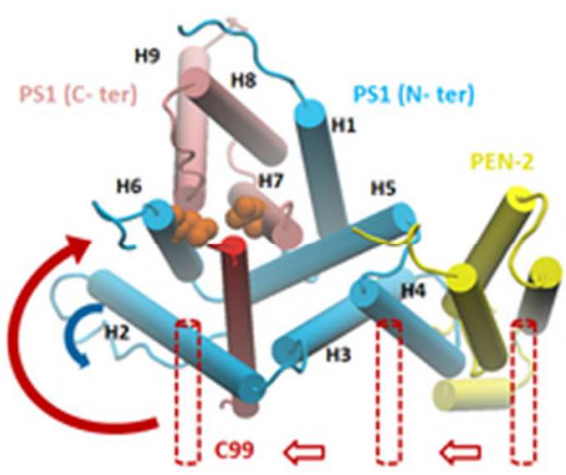

TOC figure

$18 \times 15 \mathrm{~mm}(300 \times 300$ DPI $)$ 